\title{
Development and Prototyping of Jet Systems for Advanced Turbomachinery with Mesh Rotor
}

\author{
Yuri Appolonievich Sazonov ${ }^{1}$, Mikhail Albertovich Mokhov ${ }^{2 *}$, Inna Vladimirovna \\ Gryaznova ${ }^{3}$, Victoria Vasilievna Voronova ${ }^{4}$, Khoren Arturovich Tumanyan ${ }^{5}$, \\ Mikhail Alexandrovich Frankov ${ }^{6}$, Nikolay Nikolaevich Balaka ${ }^{7}$ \\ ${ }^{1}$ Professor of Department of Machinery and Equipment for Oil and Gas Industry, Gubkin's National University of Oil and Gas, Moscow, \\ Russian Federation \\ ${ }^{2}$ Professor of Department of Oil Field Development and Operation, Gubkin's National University of Oil and Gas, Moscow, Russian Federation \\ ${ }^{3}$ Senior teacher of Department of Gas and Gas Condensate Field Development and Operation, Gubkin's National University of Oil and Gas, \\ Moscow, Russian Federation \\ ${ }^{4}$ Associate Professor of Department of Gas and Gas Condensate Field Development and Operation, Gubkin's National University of Oil and \\ Gas, Moscow, Russian Federation \\ ${ }^{5}$ Postgraduate of Department of Machinery and Equipment for Oil and Gas Industry, Gubkin's National University of Oil and Gas, Moscow, \\ Russian Federation \\ ${ }^{6}$ Junior Researcher of Department of Machinery and Equipment for Oil and Gas Industry, Gubkin's National University of Oil and Gas, \\ Moscow, Russian Federation \\ ${ }^{7}$ Head of Department of Technological Support for Wells Construction, CJSC “Russian Company for Shelf Development”, Russian Federation
}

\begin{abstract}
This article presents the research results that aim to develop promising mesh turbomachines equipped with jet control systems. The turbomachines operating in difficult conditions in oil and gas production are mainly considered. At the same time, some research results can be used in other production branches, including power engineering and transport. Three-dimensional models for computer simulation of net turbines and jet control systems were developed. Prototypes and micromodels were created to test the performance of mesh turbines and jet control systems using additive technologies. A methodological approach is proposed to create a classification of jet control systems considering their design and technological features. In the course of numerical experiments, the extreme conditions of fluid and gas outflow through a nozzle equipped with a velocity vector control system, in the control range of adjustment of the velocity vector deflection angle from $+90^{\circ}$ to $-90^{\circ}$ within a geometric hemisphere, have been considered for the first time. It was also shown that when using a dual-channel nozzle, there are possibilities to adjust the velocity vector angle (thrust vector) in the range of $+180^{\circ}$ to $-180^{\circ}$ within the geometric sphere. Compared with the known variants, the control range of the velocity vector angle is increased by nine times. These calculated data are presented in addition to the previously published results of physical laboratory experiments. Preliminary results of numerical experiments show the possibility of creating a new theory in the field of mesh turbines and jet systems. Patents support the novelty of the developed technical solutions.
\end{abstract}

\section{Keywords:}

Energy Conversion;

Energy Efficiency;

Gas Dynamics;

Hydrodynamics;

Nozzle; Ejector;

Mesh Turbine; Pump;

Article History:

Received: 25 July 2021

Revised: $\quad 18$ September 2021

Accepted: 26 September 2021

Published: $01 \quad$ October 2021

\section{1- Introduction}

In current conditions, the problem of reducing energy costs for the implementation of production processes remains relevant. In Gubkin Russian State University of Oil and Gas laboratories, a series of research works on creating new machines for working with gas-liquid mixtures-including pumps, compressors, turbines, and ejectors-have been

* CONTACT: mikhal.mokhov@mail.ru

DOI: http://dx.doi.org/10.28991/esj-2021-01311

(C) 2021 by the authors. Licensee ESJ, Italy. This is an open access article under the terms and conditions of the Creative Commons Attribution (CC-BY) license (https://creativecommons.org/licenses/by/4.0/). 
performed [1]. In these machines, flow channels have a mesh structure. In the conventional sense, a mesh represents a larger geometric area in smaller discrete cells. In a turbomachine, the larger blades are replaced by a set of smaller blades, which are interconnected to form flow channels in the form of a mesh structure. The results of studies of the mesh structure of flow channels in the development of lattice wings [2, 3], heat exchangers [4-6], and ejectors of various purposes are known [7]. In general, any ejector includes the following main parts: nozzle, mixing chamber, and diffuser. It is known that ejectors can perform the functions of a pump and a compressor; there are also known examples of using the ejector as an augmenter [8] for airborne reactive systems. The advantages of the ejector are often called the simplicity of design and the absence of moving parts. At the same time, the introduction of moving parts as part of the ejector can significantly expand the scope of such jet technology.

Possible applications of ejectors in the development of turbines and hybrid turbomachinery are possible. For example, there are known jet turbines in which the working gas is fed through a fixed inlet nozzle, and the jet coming out of the nozzle is directed along the rotor rotation axis [9]. In the central part of the rotor, there is a rotating mixing chamber with a diffuser, which provides an increase in gas pressure. Jet outlet nozzles are located at the periphery of the turbine rotor. In such turbine designs, attention may be drawn to the increased pressure in the rotor channels, especially in the section from the central diffuser to the outlet nozzle. It will entail the increase in the thickness of walls in a rotor and increase the rotor mass, with possible restrictions of rotation speed. It is also possible to note the issues of balancing axial and radial loads acting on the rotor. In addition, a jet-reactive turbine is known [10] in which the jet coming out of the nozzle is directed radially relative to the rotor rotation axis. Here, multiple mixing chambers combined into a mesh structure are used.

The formed mesh structure is placed inside the turbine rotor. In this case, nozzles and mixing chambers of unconventional forms are used, and curvilinear surfaces of flow channels help to increase the efficiency of the energy conversion process in the turbomachine. The specificity of such turbomachines is connected with non-stationary or pulse processes in the flowing part of the ejector and turbine. The simplicity of design and correspondingly low price of such turbomachines indicate the prospects of their practical use in solving urgent problems in various industries. At the same time, attention should be paid to the complexity of gas-dynamic and hydrodynamic processes occurring in the channels of such turbomachines. For example, gas flows around a set of cylinders [11], which can form a grid, are thoroughly studied now. The workflow in a mesh turbine is similar to the workflow in a partial admission turbine [12-14].

The operating processes of turbines with rotating ejectors are still poorly studied. This area of science and technology seems promising as opportunities for creating highly effective hybrid turbomachines, combining properties of jet technique and properties of impeller machines, are opened. In this regard, research of gas-dynamic and hydrodynamic processes in channels with mesh structure can be fully attributed to the number of relevant works. In aviation engineering, many problems regarding selecting the optimal geometry for solid walls in the gas flow at different flowing regimes, at high speeds $[15,16]$ and low speeds, have been solved [17, 18].

Gas flow control systems are based on mechanical systems with certain limitations on the speed of commands after the control signals are applied $[19,20]$. For improving the efficiency of technologies, it is advisable to search for new methods of gas flow control, which would differ by higher operating speeds in control systems. Prospects for the development of turbomachines are associated with a detailed study of gas flows in channels of various shapes. Increasing the efficiency of technological processes requires solving optimization problems in gas dynamics and hydrodynamics $[21,22]$.

First, a concept was developed (see Concept Headings section) to study mesh turbomachinery equipped with jet control systems. Then the materials (see Materials section) for prototyping were selected, emphasizing the application of additive technologies. In the next Methods section, a flowchart and a software package were presented, which were used for computer simulations. Technical information about the developed mesh turbines was presented in the Results section. Prototypes of the new turbine and the new jet control system were created using additive technologies. The Discussion section reflects the discussion of the presented conceptual focus area, considering the new results obtained. A new methodological approach is proposed to create a classification of jet control systems considering their design and technological features. The results of computational experiments are presented and discussed. Demonstration experiments carried out to check the performance of the created prototypes are described. The prospects for developing scientific and design works in mesh turbomachines equipped with jet control systems are considered. The discussion made it possible to summarize the research results and draw the main conclusions presented in the Conclusion section.

\section{2- Concept Headings}

The following hypothesis has been formulated: it is advisable to develop energy-saving technologies and equipment at the junction of two scientific and technical domains; the first domain is associated with mesh turbomachinery, the second domain is associated with jet control systems that allow controlling the velocity vector (thrust vector). The mesh structure of the turbomachine flow path ensures the rotor's structural strength and rigidity at a low weight. The jet control system is distinguished by high reliability (due to the small number of moving parts) at a low weight of the system as a 
whole. In this research, an intermediate goal could be the development of a jet system prototype designed to create turbomachinery with a mesh rotor. Research results primarily focus on creating multi-mode turbomachines operating in difficult conditions (including in the conditions of offshore oil and gas fields). Simultaneously, the research results can also be used to solve practical problems in other industries, including increasing the efficiency of energy conversion processes to generate electrical energy.

Our publications have already presented the results of physical experiments carried out in laboratory conditions. The question arises about the need for a partial transition from physical experiments to numerical studies, which will save time and material resources. It is important to confirm the possibility of jet diaphragm deflection by 90 degrees using computer simulations. In the course of a physical experiment, it was previously proved that the jet could undergo diaphragm deflection by 90 degrees $[1,23,24]$. This paper presents new research results and describes numerical experiments, confirming the possibility of jet deflection by 90 degrees. It is shown that numerical experiments can partially replace expensive physical experiments. The computer simulation results reflect the main flow patterns at the outlet of a nozzle equipped with a diaphragm.

The main research tasks at this stage include 1) the development of gas flow control principles in the channels of the mesh turbine, 2) the development of a jet control system prototype for the mesh turbine, and 3) confirm the possibility of jet diaphragm deflection by 90 degrees using computer simulation.

\section{3- Materials and Methods}

\section{3-1-Materials}

3D printers were used to manufacture prototypes (Figure 1a); the characteristics of these printers are given in Table 1. The purpose of the prototypes is marked in the flowchart (Figure 1b).

Table 1. Printer characteristics.

\begin{tabular}{ccc}
\hline 3D Printer model & ZAV-L & Ultimaker 2 (RepRap) \\
\hline Manufacturing country & Russia & Russia \\
Plot area $(\mathrm{X} \times \mathrm{Y} \times \mathrm{Z}), \mathrm{mm}$ & $180 \times 200 \times 180$ & $200 \times 200 \times 200$ \\
Maximum print speed, $\mathrm{mm} / \mathrm{s}$ & 120 & 90 \\
Nozzle diameter, $\mathrm{mm}$ & 0.3 & 0.6 \\
Extruder maximum temperature, ${ }^{\circ} \mathrm{C}$ & 280 & 280 \\
Maximum heated bed temperature, ${ }^{\circ} \mathrm{C}$ & 150 & 150 \\
Extruder type & Bowden & Bowden \\
Positioning accuracy, mm & 0.02 & 0.02
\end{tabular}

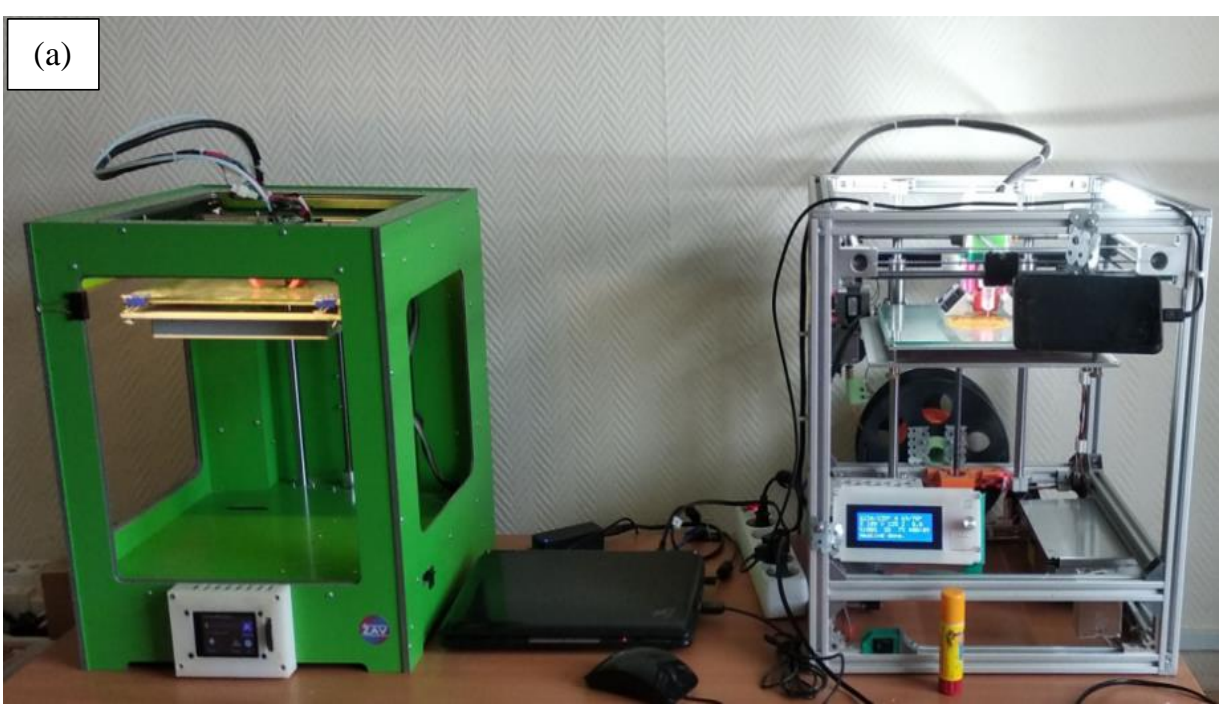


The main concept is related to the study of gas-dynamic and hydrodynamic processes in the pulse mode of flow through rotating and fixed channels with a mesh structure

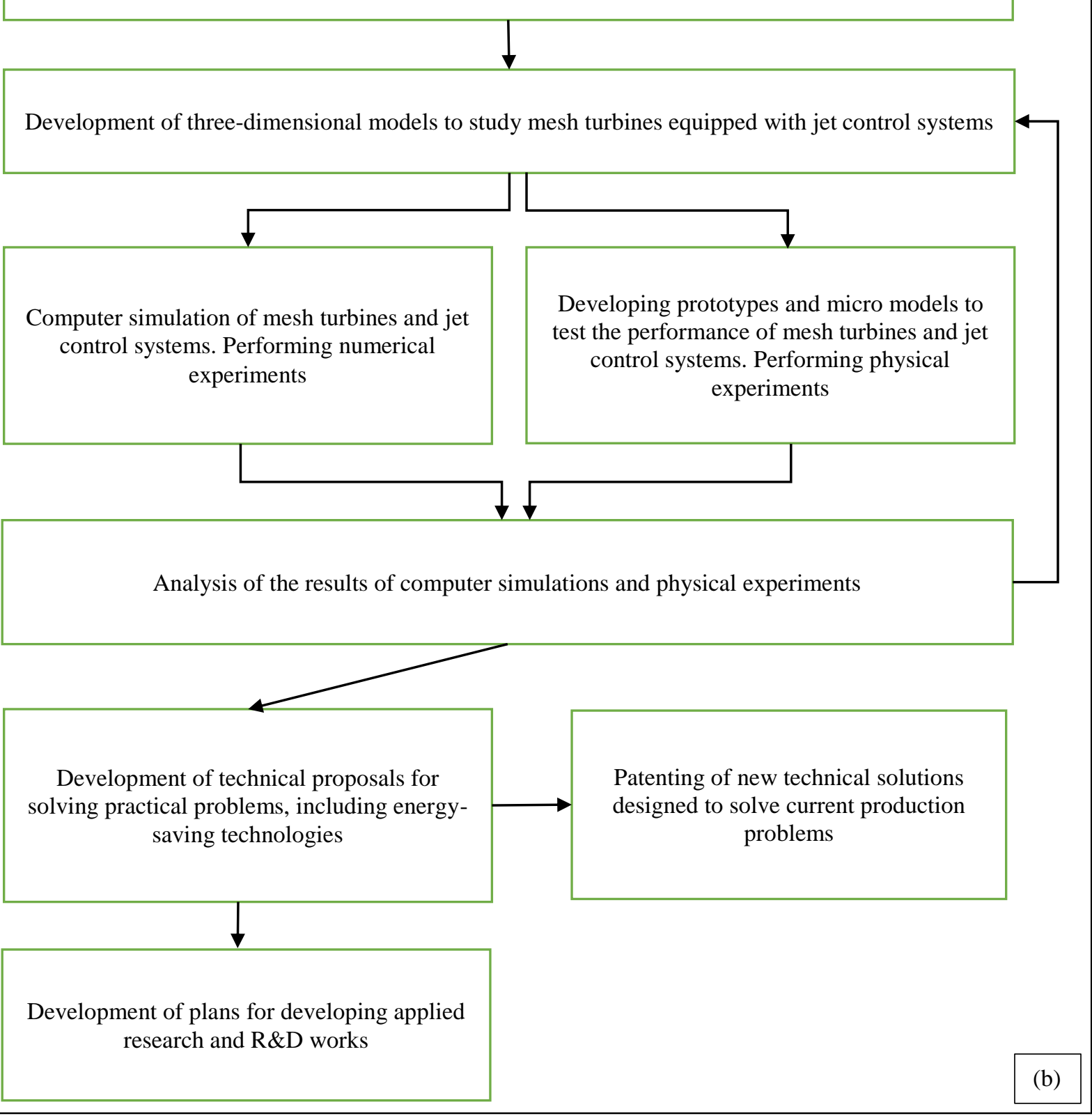

Figure 1. (a) Photo of 3D printers used to develop mesh turbines with jet control systems and (b) flowchart to explain the research methodology.

\section{3-2-Methods}

The software package Flow Simulation (FloEFD) was used for computer simulation and computational research. The 3D model was created using the SolidWorks 3D CAD system. In the process of modeling, a complete system of NavierStokes equations - described by mathematical expressions of the laws of mass, energy, and momentum conservationwas solved. The $k-\varepsilon$ turbulent viscosity model was used.

CPU type: Intel(R) Core (TM) i5-6200U CPU@2.30GHz; CPU speed: $2401 \mathrm{MHz}$; RAM: 8065 MB; Operating system: Windows 10 .

The main concept is related to the study of gas-dynamic and hydrodynamic processes during pulsed flow through rotating and fixed channels with a mesh structure (Figure 1b). The development of three-dimensional models for the study of net turbines equipped with jet control systems is envisaged. Based on the three-dimensional models, computer simulations of the mesh turbines and jet control systems are performed. In parallel with the calculations, prototypes and micro models are created to test the performance of the mesh turbines and jet control systems. Additive technologies are used for this purpose. Based on the results of numerical and physical experiments, technical proposals are developed to 
solve practical problems, including the creation of energy-saving technologies. The research results are also used for creating new models and prototypes and as a basis for future applied research and R\&D works. Separate developments are patented.

\section{4- Results}

New approaches to developing special turbines, pumps, and compressors are being considered part of the ongoing comprehensive research. The peculiarity of such machines is using a mesh structure in profiling the rotor and stator flow parts in a hydraulic machine of the dynamic type. The possibilities for the practical use of the ejector are also taken into account, given the rotational motion of its separate parts. When developing a new turbine, known designs, including a patent [25], were considered. The disadvantage of this turbine is that a quick (or emergency) rotor stop will require correspondingly quick working flow overlapping that can provoke a hydraulic shock. The technical problem to be solved with the new turbine design is strengthening control over rotor speed under operating conditions (and under extreme conditions) by reducing time to stop the rotor (deceleration). That increases the turbine operation safety and expands the application area. The achievable technical result is the prevention of pressure pulsations and hydraulic shocks during rotor braking and stopping. It is achieved by flow control at the outlet of nozzle diaphragms. The essence of the developed turbine [26] is explained in the figures. Figure 2 shows a schematic of the mesh turbine, Figure $3-$ its A-A cross-section with the open nozzle. Figure 4 presents its $\mathrm{A}-\mathrm{A}$ cross-section in the rotor braking and stopping mode, Figure 5 - the same with the closed nozzle. Figure 6 shows a schematic of a control sleeve with through holes, and Figure 7 shows a B-B cross-section of the mesh turbine when using a rotary hydraulic cylinder.

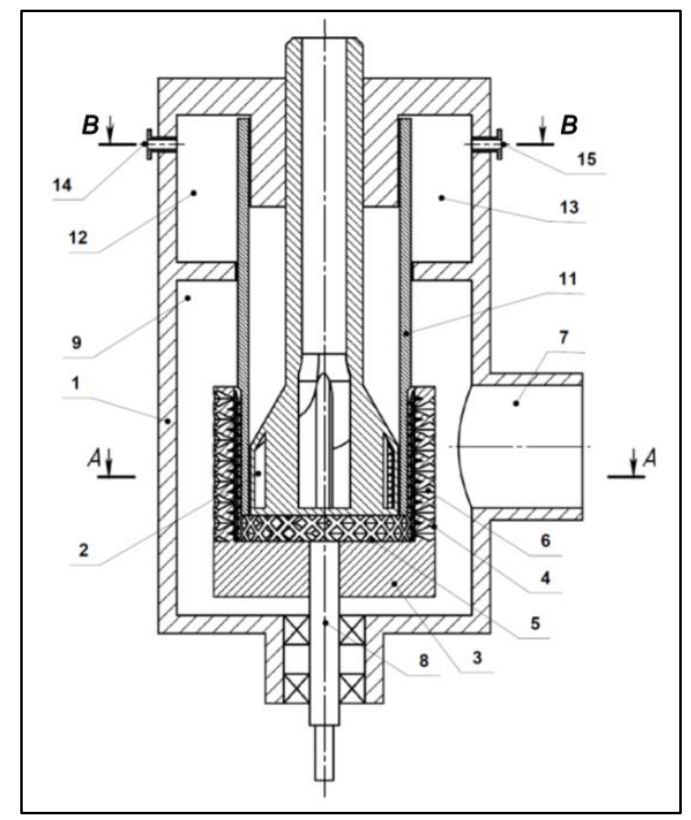

Figure 2. Schematic of the mesh turbine: 1- stator; 2- inlet nozzles; 3- rotor; 4- outer protrusions; 5- inner protrusions; 6- flow channels; 7- outlet channel; 8- rotation axis; 9- vortex chamber; 10- circulating annular channel; 11- movable control sleeve; 12 and 13- hydraulic chambers; 14 and 15- through sockets; 16- through-holes.

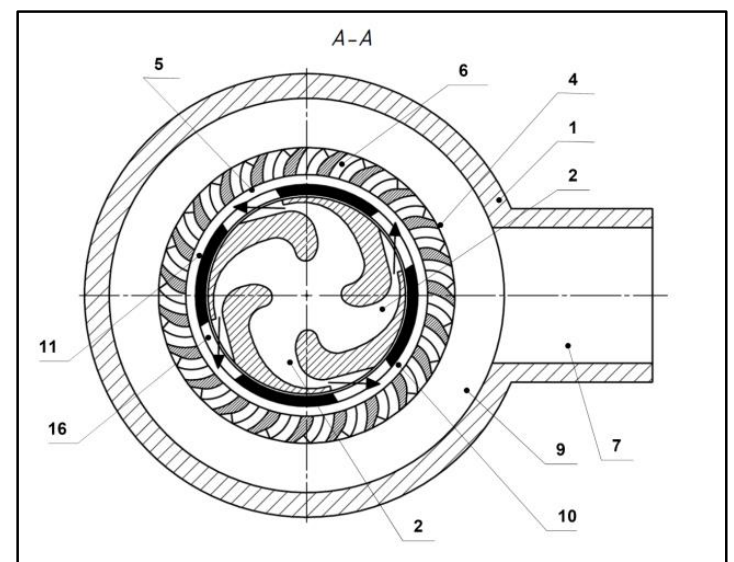

Figure 3. Schematic of the mesh turbine, A-A cross-section with the open nozzle: 1- stator; 2- inlet nozzles; 4- outer protrusions; 5- inner protrusions; 6- flow channels; 7- outlet channel; 9- vortex chamber; 10- circulating annular channel; 11-movable control sleeve; 16- through-holes. 


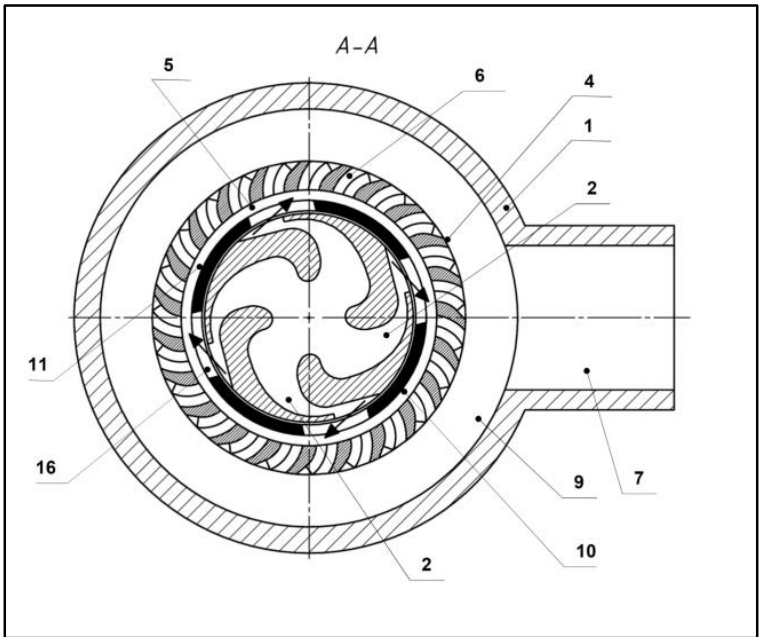

Figure 4. Schematic of the mesh turbine, A-A cross-section in the rotor braking and stopping mode.

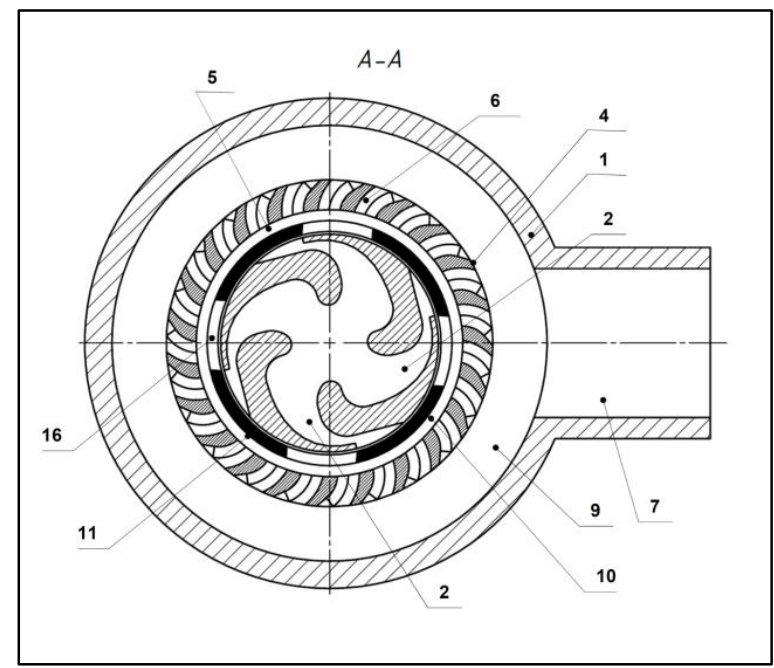

Figure 5. Schematic of the mesh turbine, A-A cross-section with the closed nozzle.
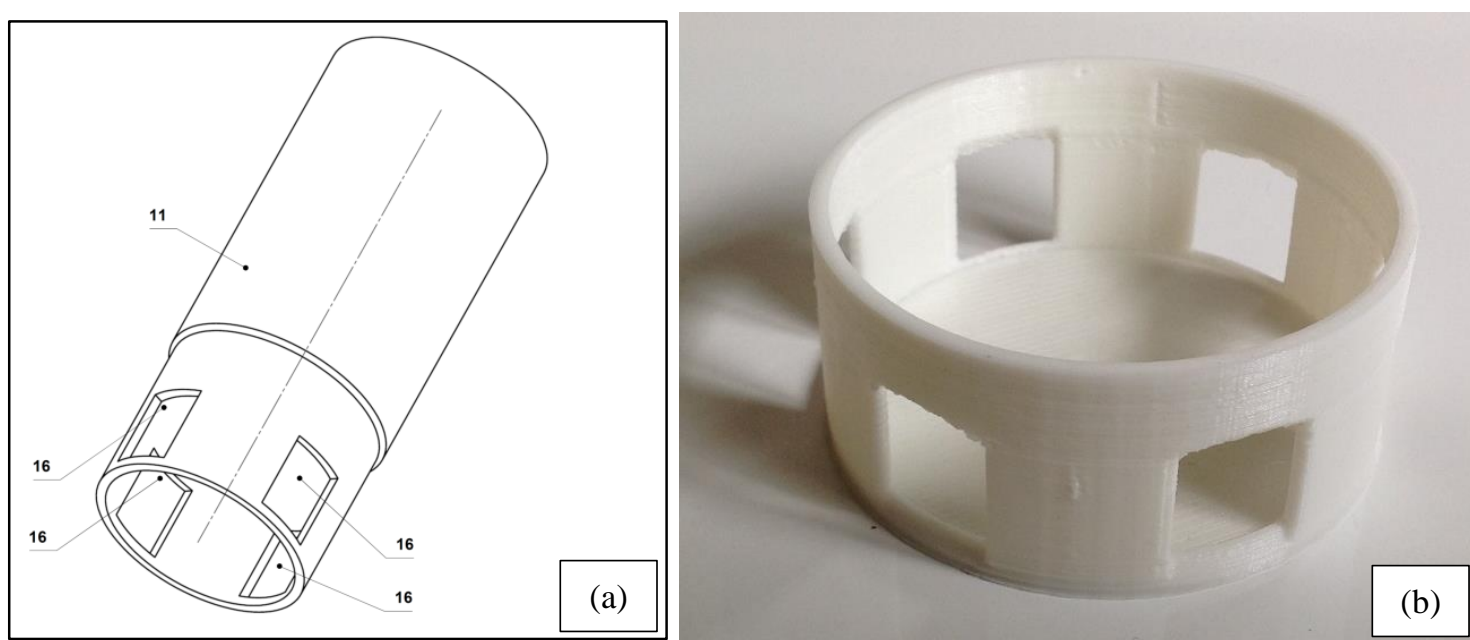

Figure 6. Schematic of the control sleeve (meshed diaphragm) with through holes: (a) and a prototype; (b) 11- movable control sleeve; 16- through-holes.

Figure 6 shows a meshed diaphragm with holes on a cylindrical surface. Flat diaphragms [27] are known to control the flow at the nozzle outlet. It is also possible to use a spherical surface to make a diaphragm with multiple holes for the passage of gas or liquid. The holes themselves may be a circular, square, rectangular, rhombus, or other geometry, including unique variants with unconventional geometry. The unconventional nozzle and methods of regulation of such nozzle diaphragms are still very poorly understood. 


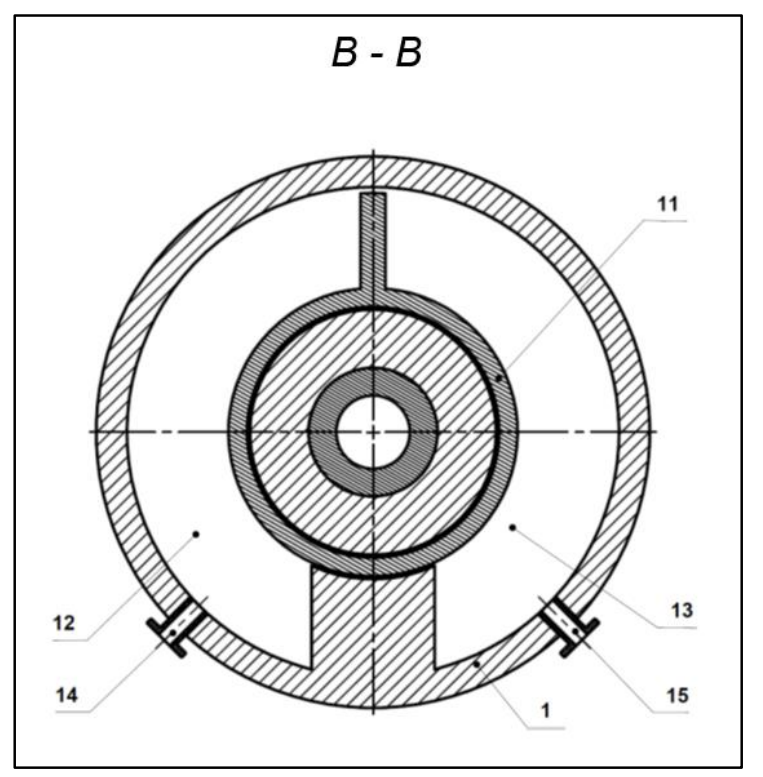

Figure 7. B-B cross-section of the mesh turbine using a rotary hydraulic cylinder: 1- stator; 11- movable control sleeve; 12 and 13- hydraulic chambers; 14 and 15- through sockets.

The developed turbine contains stator 1 with inlet nozzles 2 and is placed in it rotor 3 with outer protrusions 4 . The turbine design can have one, two, or more nozzles. The nozzle diaphragm may have a mesh structure, where the surface of the nozzle has many holes. Rotor 3 is equipped with inner protrusions 5 and comprises a porous mesh structure consisting of protrusions 4 and 5 interconnected and having solid walls. Between inner 5 and outer 4 protrusions in rotor 3 , there are flow channels 6 , providing hydraulic connection of flow channels 6 in rotor 3 with inlet nozzles 2 in stator 1. Stator 1 is equipped with outlet channel 7. Given the turbine application conditions, inlet nozzles 2 and outlet channel 7 can be located at different distances from rotation axis 8 of rotor 3 .

Stator 1 contains vortex chamber 9 installed coaxially with the rotor. Inlet nozzles 2 are located in the central part of rotor 3, forming circulating annular channel 10 in the gap between inlet nozzles 2 and inner protrusions 5 . In vortex chamber 9, movable control sleeve 11 is placed with the possibility of adjusting its adjustable rotary movement for partial or complete overlapping of inlet nozzles 2. The position of movable control sleeve 11 can be changed using known technologies, such as a hydraulic or electromagnetic drive. The figures show a variant with a hydraulic drive to rotate movable control sleeve 11. Hydraulic chambers 12 and 13 can be connected to the hydraulic control system through sockets 14 and 15 (the hydraulic control system is not shown in the figures). Control sleeve 11 has throughholes 16 through which inlet nozzles 2 communicate with flow channels 6 of rotor 3 . Inflow channels 6 of rotor 3 can implement the ejector workflow, and flow channels 6 can act as a mixing chamber and ejector diffuser. The presented variant uses many mixing chambers combined into a mesh structure. The formed mesh structure is placed inside the turbine rotor. In this case, nozzles and mixing chambers of unconventional shape are used. The curvilinear surfaces of the flow channels help increase the efficiency of the energy conversion process in the turbomachine. The developed design of the turbine with a jet control system is patented in Russia [26].

The turbine works as follows:

Stator 1 and inlet nozzles 2 form the flow (or several flows) of the fluid (medium) towards rotor 3 (Figure 2). The medium can be liquid, gas-liquid mixture, or gas (including steam or high-temperature combustion products of fuel-air mixture). The fluid flow impacts on inner protrusions 5 of rotor 3 and puts rotor 3 in motion. Thus, the kinetic energy of the fluid flow is converted into mechanical energy during the rotary motion of rotor 3 . Rotor 3 can be connected with external mechanisms (not shown in the figures).

In addition, the fluid flow through flow channel 6 penetrates the cavity of rotor 3 , presented as a porous volumetric mesh structure. The fluid flow in this part of rotor 3 interacts with the inner 5 and outer 4 protrusions' solid walls, contributing to energy conversion. It is possible to implement both continuous and pulse fluid flow to the turbine rotor 3 . The permeable mesh structure of rotor 3 provides energy conversion at different fluid properties, including gas-liquid mixtures different in density or viscosity. The fluid is removed through flow channel 6 in rotor 3 and outlet channel 7 out of stator 1 .

The position of movable control sleeve 11 and accordingly the one position of through-holes 16 can be changed. To close inlet nozzles 2, hydraulic chamber 12 is supplied with liquid under high pressure through socket 14 . The liquid is displaced from hydraulic chamber 13 into the low-pressure line through nozzle 15. Under the differential pressure in the hydraulic chambers 12 and 13, movable control sleeve 11 turns around rotation axis 8 and closes nozzles 2. 
For opening inlet nozzles 2, the liquid is supplied into hydraulic chamber 13 under high pressure through socket 15 . As a result, the fluid is displaced from hydraulic chamber 12 into the low-pressure line through socket 14. Owing to the pressure difference between hydraulic chambers 12 and 13, movable control sleeve 11 turns around the rotation axis 8 , opening inlet nozzles 2. It is worth noting that the intermediate position of movable control sleeve 11 can be changed by modifying the volume of liquid contained in the two hydraulic chambers (which is a common approach in hydraulically-driven control systems based on a rotary hydraulic cylinder, as shown in Figure 7).

Moreover, due to the angular movement of movable control sleeve 11, the flow rate at the outlet of nozzles 2 can be regulated through their partial or complete overlap, which would, in turn, modify the turbine working mode. In addition, by changing the position of through-holes 16 relative to nozzles 2, it is technically possible to reverse the rotation direction of rotor 3, as shown in Figure 4.

Figure 3 shows the A-A cross-section of the turbine with nozzles 2 in the open position, with the flow direction at their outlet indicated by arrows. As can be seen from the image, turning movable control sleeve 11 in a clockwise direction can arrive at the position shown in Figure 4, thereby changing the flow direction at the outlet of nozzles 2 as indicated by the arrows. Due to the pressure-induced by medium flow, rotor 3 will gradually decelerate until it reaches a complete stop, even though the medium can flow towards outlet channel 7 . In contrast to the turbine under the patent [25], the new turbine [26] has new design features and technical capabilities. Nonetheless, stopping rotor 3 will prevent pressure pulsations and hydraulic shocks when braking. In other words, rotor 3 will cease to move whether the inlet nozzles 2 are completely (Figure 5) or partially (Figure 4) closed. Therefore, the energy of the medium flow helps strengthen the control of the rotor speed when operating in extreme modes while reducing its stopping time (expediting its deceleration). This design feature increases the turbine operation safety and expands the range of its applications, including production facilities in the oil and gas industry.

The developed operating principle of the turbine [26] was considered through an example, which does not limit the scope of application of the new technical solution. At the same time, turbine modifications are possible within the framework of the disclosed invention [26]. Based on the presented principle of operation, it is possible to create various single-stage and multi-stage impeller machinery with a mesh structure of flow channels. The jets can move in the radial and the axial direction. For illustration, Figures 8-14 show photos of the developed and manufactured impeller prototypes for mesh centrifugal and axial turbines and a nozzle apparatus prototype operating according to the described principles under the patent [26].

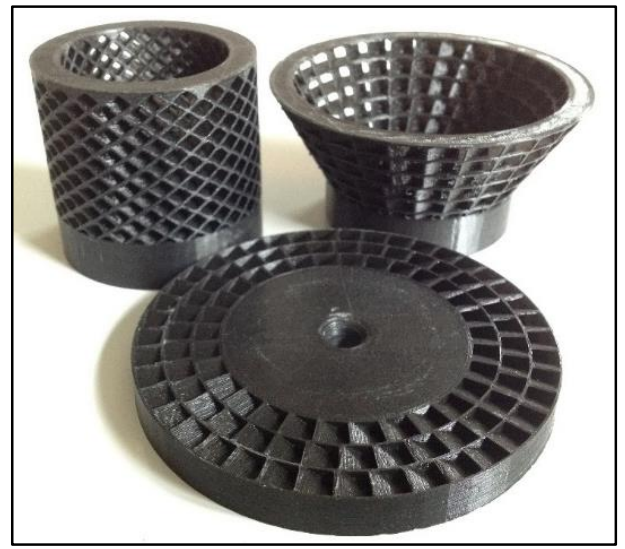

Figure 8. Mesh turbine impeller prototypes: on the left in the background - a cylindrical centrifugal turbine, in the foreground in the center - an axial disk turbine, on the right in the background - a conical centrifugal turbine.

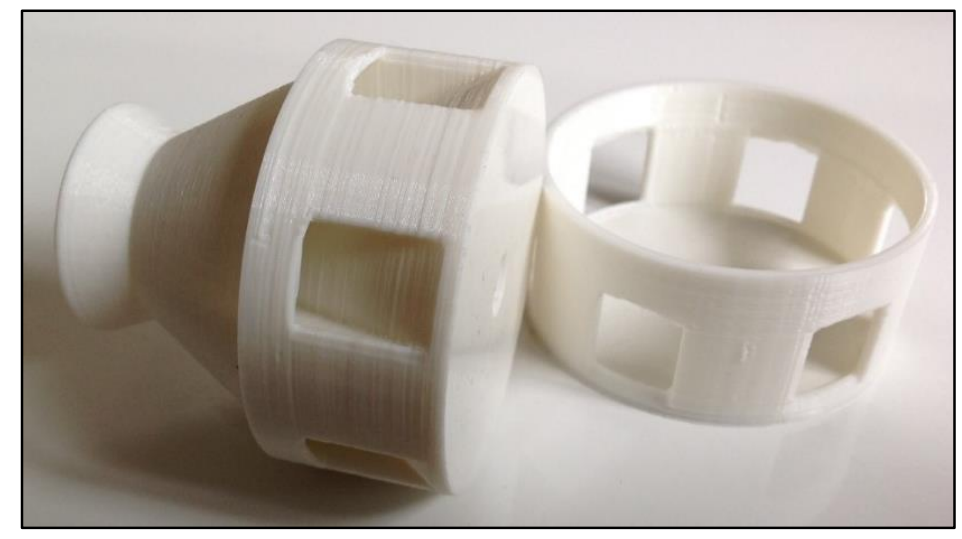

Figure 9. A nozzle block prototype (shown on the left) and a control sleeve (diaphragm) prototype. 


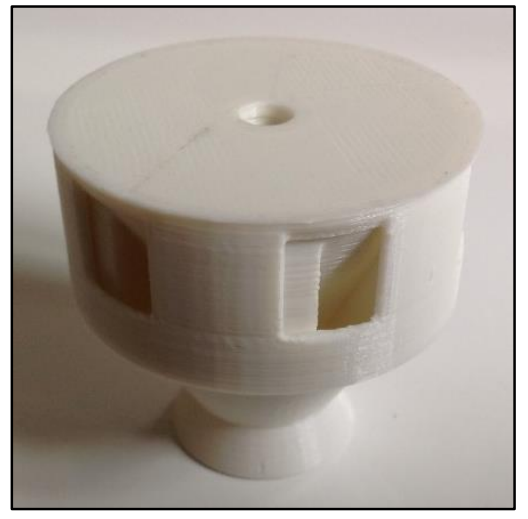

Figure 10. A nozzle block prototype (shown on the left) and a control sleeve (diaphragm) prototype in the working position.

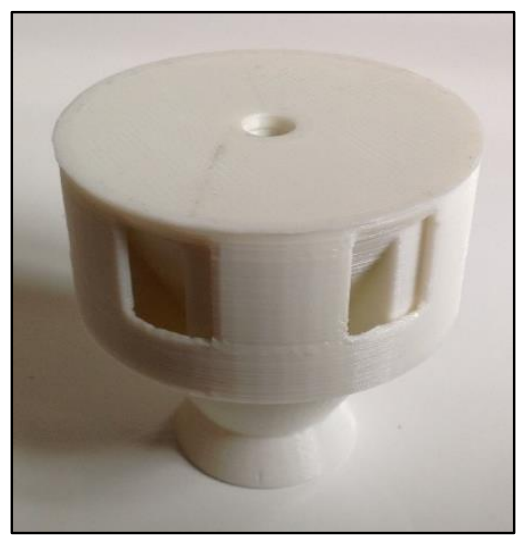

Figure 11. A nozzle block prototype (shown on the left) and a control sleeve (diaphragm) prototype in the position for reversible rotation of the turbine impeller.

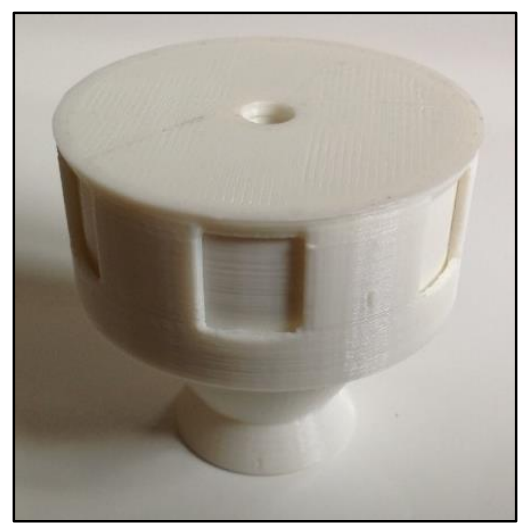

Figure 12. A nozzle block prototype (shown on the left) and a control sleeve (diaphragm) prototype in the closed position.

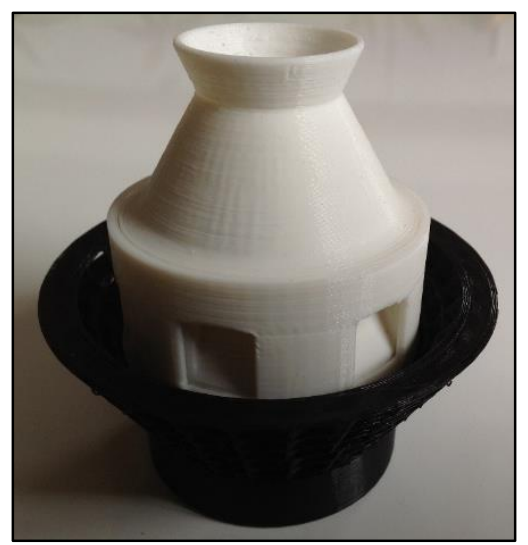

Figure 13. An impeller, nozzle, and control sleeve (diaphragm) prototype together with a conical centrifugal turbine prototype. 


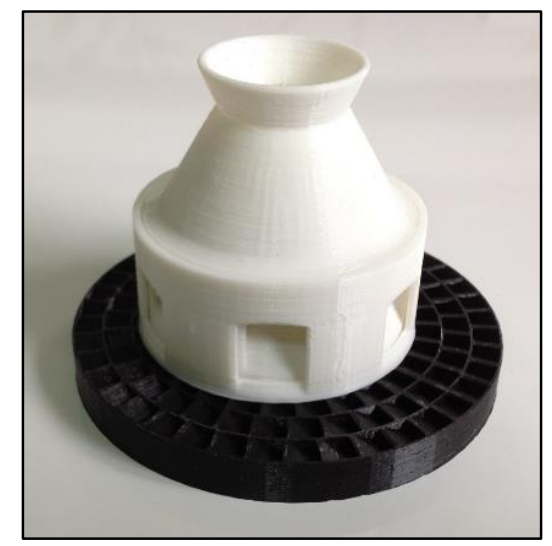

Figure 14. An impeller, nozzle block, and control sleeve (diaphragm) prototype together with a disk axial turbine prototype.

The turbine developed and presented in this article is similar to a partial admission turbine [12-14]. As a rule, a turbine is called a partial admission one when the nozzle block has blades only on the part of its circumference; in this connection, the gas is supplied to the impeller only within this part of the circumference. Figure 15 shows a typical schematic diagram of a partial admission turbine.

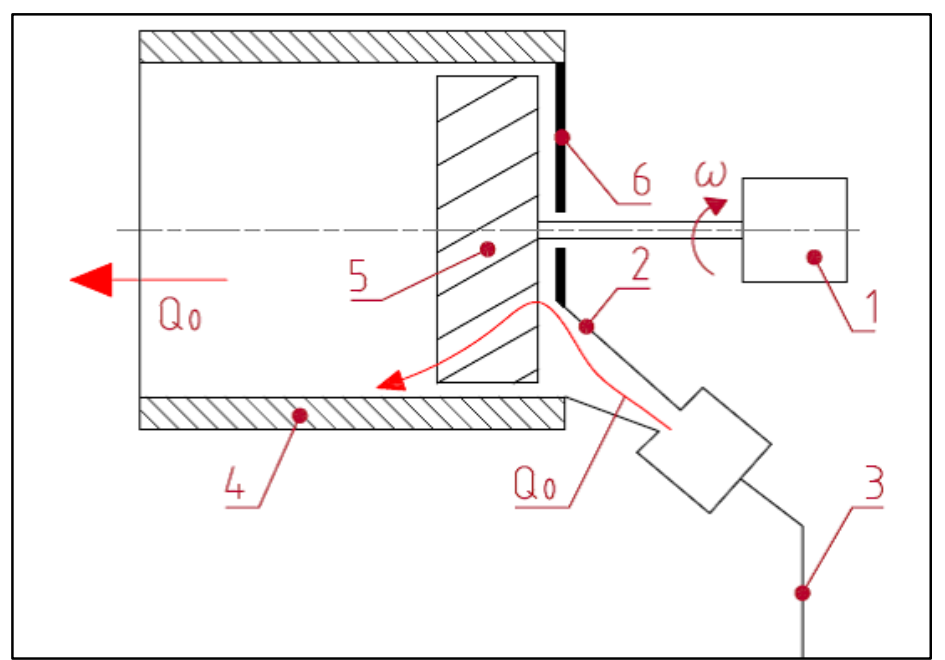

Figure 15. Schematic of a partial admission turbine: 1- generator; 2- nozzle block; 3- working gas source; 4- casing; 5- impeller; 6- separation disc.

In this case, the energy conversion process during the jet interaction with the solid walls of the impeller can be considered separately (Figure 15). When discussing the principle of operation, it is possible to use the following simplified formula for a partial admission turbine.

$N_{0}=N_{m}+d N$,

where $N_{0}$ is the working fluid flow rate with the mass flow rate $Q_{0} ; N_{m}$ is mechanical power transmitted through the shaft connecting impeller 5 to the generator $1 ; d N$ is power loss in impeller 5 . The efficiency of such an energy conversion process for a partial turbine can be expressed using multiple valuation $\eta_{x}$.

$\eta_{x}=\frac{N_{m}}{N_{0}}$

Suppose the separation disk 6 is removed from the partial admission turbine, and the nozzle block is equipped with a velocity vector (or thrust vector) control system. In that case, it is possible to pass on to a new class of impeller machinery. At the discussion stage, this new class of machines will be called hybrid mesh machinery. Figure 16 shows a schematic diagram of a hybrid mesh turbine.

The velocity vector (thrust vector) control system can have various embodiments [25]. This paper considers a variant of controlling the velocity vector using a movable diaphragm installed at the nozzle outlet. A diaphragm with a round hole can be called a classic one. In the developed prototype and the patent [26], a diaphragm with several rectangular holes made on a cylindrical wall is used; all these diaphragm embodiments operate on the same principles. 

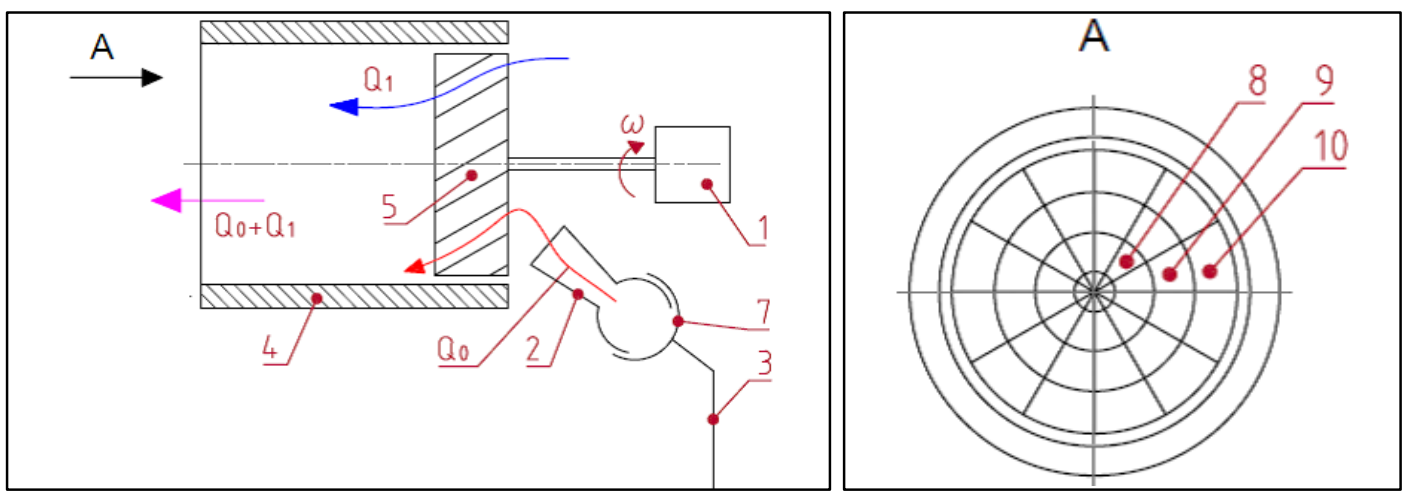

Figure 16. A hybrid mesh turbine schematic: 1- generator; 2- nozzle block; 3- working gas source; 4- casing; 5- impeller; 7- velocity vector control system; 8,9 , and 10 - annular flow channels in which the blades are located.

The velocity vector control system 7 enables direct the working fluid flow into the annular channel 8, 9, or 10, solving the technical problem (view-A in Figure 16). In this case, it is possible to regulate the torque on the impeller shaft since the working radius changes (the distance from the impeller rotation axis to the point at which the working fluid flow interacts with the solid walls of the impeller). The blades in the annular flow channels 8,9 , and 10 can have different geometry and sizes, depending on the technical or technological problem. In a hybrid mesh turbine, two different workflows can run simultaneously. The first process is a turbine workflow, which is similar to the workflow taking place in the partial turbine channels. The second workflow is a pumping process if the pumped fluid is a liquid. If the pumped fluid is a gas, the second workflow can be a compressor or fan process, depending on the pressure difference at the impeller inlet and outlet. A variant is also considered when the pumped fluid is a multiphase medium (gas-liquid mixture, for example). In the diagram, the part of the impeller located below the rotation axis participates in implementing the turbine workflow (or the first workflow). The kinetic energy of the working fluid flow $Q_{0}$ is converted into mechanical energy, which is transferred to the impeller. In the diagram, the part of the impeller located above the rotation axis participates in implementing the second workflow. The impeller blades exert a force effect on the pumped fluid, transferring part of the energy to create (form) the flow $Q_{1}$.

A more detailed examination of the hybrid turbine requires considering separately the third working process, which takes place behind the impeller, in casing 4 . Here, the ejector workflow will be implemented. The process of mixing the working fluid flow $Q_{0}$ with the pumped medium flow $Q_{1}$ can be fully considered as a separate process, namely, as an ejector workflow. Casing 4 serves as a mixing chamber within the ejector working process. Since impeller 5 is located in casing 4 between the nozzle block and the outlet channel, it can be stated that the flow channels of impeller 5 are part of the mixing chamber. The flow channels of impeller 5 make a rotational movement, activating the energy conversion process and activating the ejector workflow. Thus, it is necessary to suggest an ejector of an unconventional shape, in which the mixing chamber consists of at least two parts. In this case, one of these two parts of the mixing chamber makes a rotational movement. Such ejectors of unconventional shape can act as objects for independent scientific research. Considering the variety of applied (practical) tasks, we can discuss the expediency of developing this scientific domain. For example, certain tasks and operating conditions are known, in which the ejector operates with low efficiency, at the level of $15 \%$. In the future, it will be possible to create an unconventional ejector with higher efficiency, at the level of $30 \%$ for these conditions.

The energy conversion process can be separately considered for a hybrid mesh turbine when the jet interacts with the solid walls of the impeller (Figure 16). At the stage of discussing the principle of operation, the following simplified formula can be applied:

$N_{0}=N_{m}+N_{1}+d N$,

Where $N_{1}$ is the flow rate of the pumped medium with the mass flow rate $Q_{1}$. Good yield in such a simplified system can be estimated by the sum of two terms $\left(N_{m}+N_{1}\right)$. The efficiency of such an energy conversion process for a hybrid turbine can be expressed using multiple valuation $\eta_{x}$.

$\eta_{x}=\frac{N_{m}+N_{1}}{N_{0}}$

Figure 17 shows a schematic diagram of a hybrid mesh turbine when switching between operating modes. The velocity vector control system enables to direct part of the working fluid or the entire flow 11 in the other direction, bypassing impeller 5 . This switching may be associated with the need for the turbine emergency shutdown for safety reasons. Alternatively, such switching may be related to solving other problems within the framework of controlling the entire technological system, in which the hybrid turbine is considered a subsystem. 


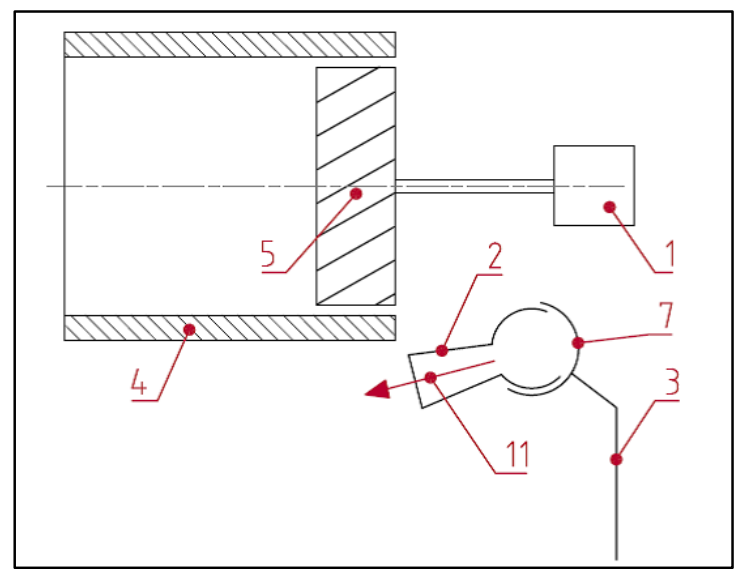

Figure 17. Schematic of a hybrid mesh turbine when switching the operating mode: 1- generator; 2- nozzle block; 3- working gas source; 4- casing; 5- impeller; 7- velocity vector control system; 11- working fluid flow.

The considered basic principles of a hybrid mesh turbine operation can be used to develop new technical and technological systems in various industries. For example, another machine can be used instead of generator 1 in Figure 16, such as a pump, a fan, or a compressor. Alternatively, another motor can be used instead of generator 1 in Figure 16: an electric motor or a turbofan gas turbine engine. In this case, impeller 5 of Figure 16 will operate as an afterburner for a temporary increase in power and thrust; such prospects are already visible for unmanned vehicles.

\section{5- Discussion}

When developing new jet systems in this study, research results from various industries, including aviation and space technologies, are utilized to expedite the process. In this regard, the study of jet technology has considered the control of the velocity vector or thrust vector, using aviation terminology. That is a novel approach to addressing this issue, as hydrodynamic and gas-dynamic systems' scientific and technical potential in controlling processes associated with changes in the fluid or gas flow direction, including the consideration of magneto-hydrodynamic control systems [28], has not been fully explored. As was shown in this work, the application area of new turbomachines can be greatly expanded by finding suitable means of changing the direction of fluid or gas flow.

Figure 18 shows a schematic diagram of the switchgear variant with the ability to change (regulate) the direction of one or two flows. Additionally, a 3D model is shown for the switchgear variant, which explains the principles of flow distribution. In this example, a flow of liquid or gas is supplied to switchgear 1 through inlet channel 2. At the outlet of switchgear 1, one or two jets (denoted by 3 and 4 ) are formed, flowing into the surrounding space that can be filled with liquid or gas. The schematic diagram shows axial line 5 and angles $\alpha$ and $\beta$ denoting jet direction. This angular parameter can be called the deflection angle of the velocity vector (or the deflection angle of the thrust vector, by analogy with the term used in aviation technology).
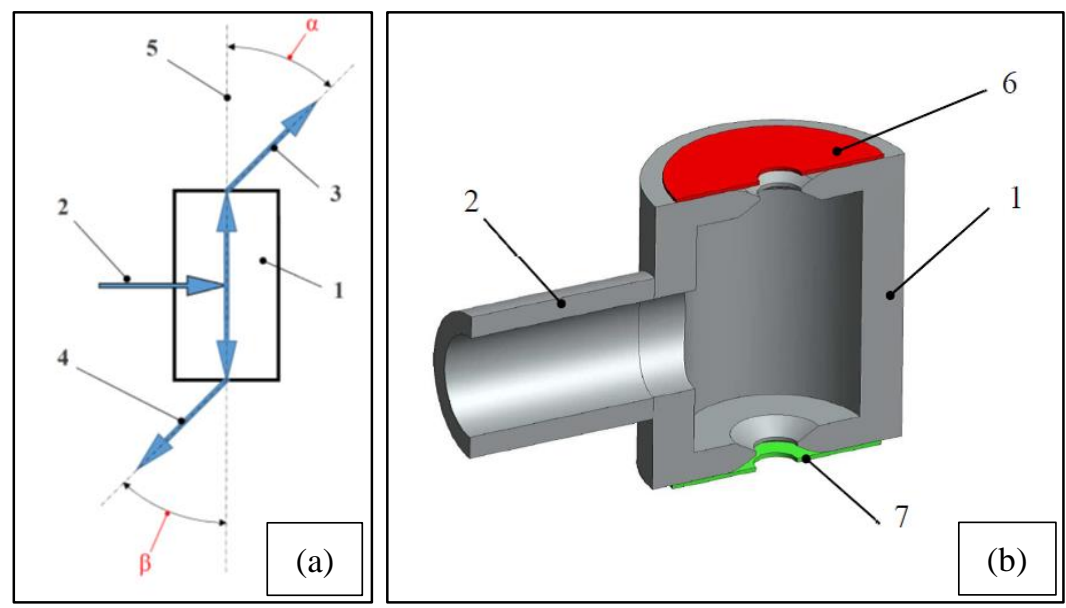

Figure 18. Switchgear diagram - variant No. 1 and a 3D model: 1- switchgear; 2- inlet channel; 3 and 4- gas jet; 5- axial line; 6- upper movable diaphragm; 7- the bottom movable diaphragm.

Radial displacement of diaphragms 6 and 7 changes the angles " $\propto$ " and " $\beta$ ". In extreme positions, the diaphragm can completely block the flow channel. 
According to the available evidence about known control systems, the deflection angle of the thrust vector (denoted $\propto$ in Figure 18) can vary from $+20^{\circ}$ to $-20^{\circ}[25,29,30]$. However, laboratory test results $\propto$ can take any value from $+90^{\circ}$ to $-90^{\circ}$ in any direction (i.e., within a hemisphere) [1, 23].

These findings suggest that the control range of the thrust vector deflection angle can be significantly extended. Additionally, note that the control range can be extended even further with a more comprehensive approach to design. When designing multi-mode and universal turbine technologies, the goal to achieve an extremely large control range for changing $\propto$ from $+180^{\circ}$ to $-180^{\circ}$ in all directions have been set. Preliminary design studies and laboratory tests of the micromodels have shown that this goal is achievable and $\propto$ can vary from $+180^{\circ}$ to $-180^{\circ}$ in any direction within a full three-dimensional sphere, for example, in simultaneous regulating the mass rate of gas and $\propto$ and $\beta$ values for two jets. More detailed information on this issue is planned to publish after conducting additional patent research. There is a good prospect for improving the technical performance of such control systems, and scientific and patent research should continue in this direction. Table 2 shows a schematic representation of the switchgear, indicating the flow direction for some working modes, given the similarity with the diagram in Figure 18.

Table 2. Switchgear working modes.

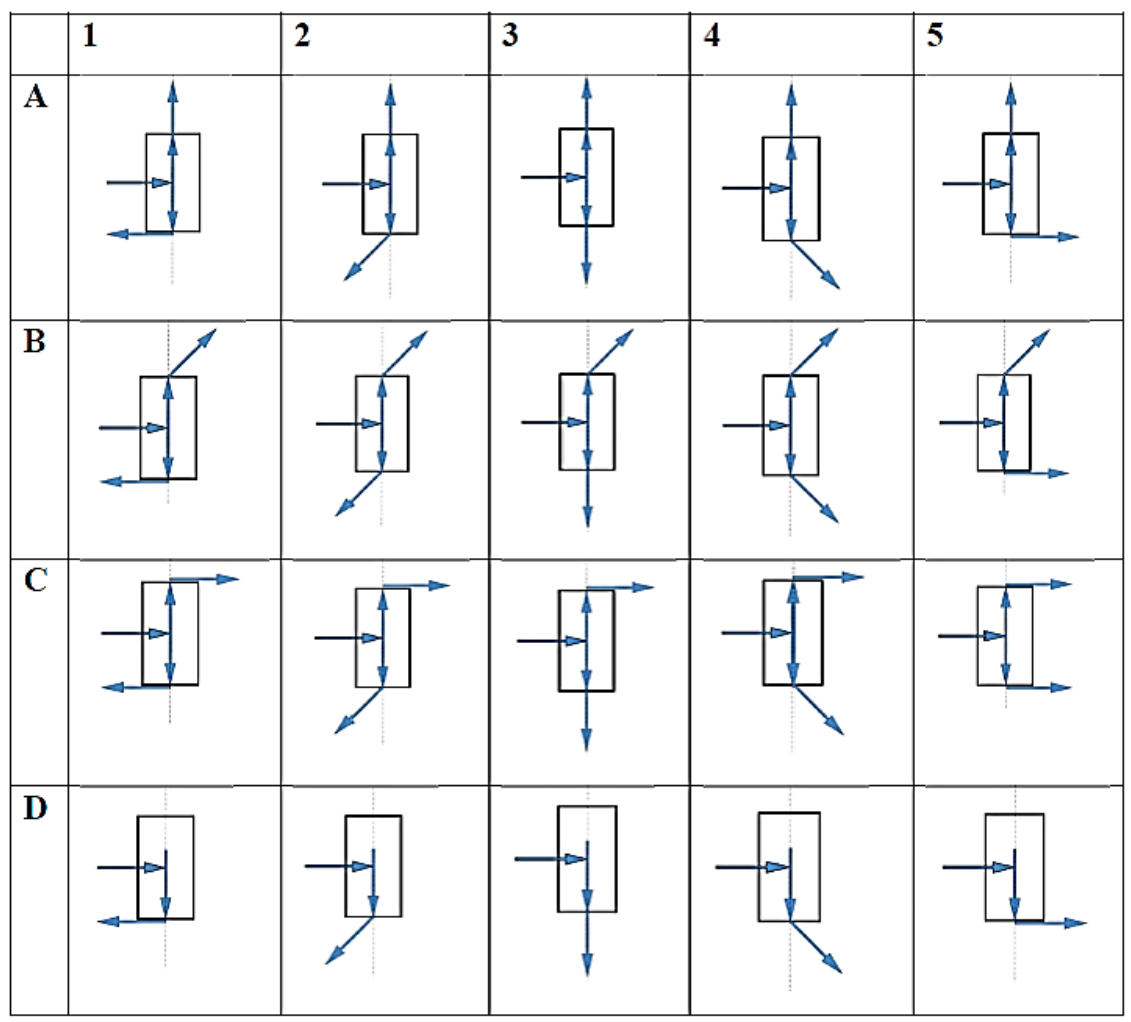

In Table 2, line "A" schematically shows the variants in which angle $\propto=0$. The gas flow rate through the upper channel $Q_{\alpha}>0$. Through the lower channel $-Q_{\beta}>0$. In this case, angle $\beta$ can vary over a wide range of values ($90) \leq \beta \leq(+90)$. Line "B" schematically shows some variants in which $\propto$ can vary over a wide range of values ($90) \leq \propto \leq(+90)$, the gas flow rate through the upper channel $Q_{\propto}>0$. Through the lower channel $-Q_{\beta}>0$. In this case, $\beta$ can also vary in a wide range of values $(-90) \leq \beta \leq(+90)$. Line " $C$ " schematically shows some variants in which $\alpha=(+90)$, the gas flow rate through the upper channel $Q_{\propto}>0$. Through the lower channel $-Q_{\beta}>0$. In this case, $\beta$ can vary in a wide range of values: $(-90) \leq \beta \leq(+90)$. Line " $D$ " schematically shows some variants in which the gas flow rate through the upper channel $\mathrm{Q}_{\propto}=0$. Through the lower channel $-Q_{\beta}>0$. In this case, $\beta$ can vary in a wide range of values: ($90) \leq \beta \leq(+90)$. By analogy, other variants can be considered when $Q_{\propto}>0$. And the gas flow rate through the lower channel $Q_{\beta}=0.1$.

Figure 19 shows a schematic diagram of the switchgear variant to change (regulate) the flow direction: the gas flow rate through the upper channel $Q_{\propto}>0$, through the lower channel $-Q_{\beta}=0$. In this example, the liquid or gas flow is supplied to switchgear 1 through inlet channel 2 . At the outlet of switchgear 1 , one jet 3 is formed, flowing out into the surrounding space. The diagram shows axial line 5 and angles $\propto 1$ and $\propto 2$ to quantify the jet direction. Jet 3 can interact with the solid wall of deflector 4 , which can be stationary or movable. This paper considers the variant in which deflector 4 can perform a rotational motion around rotation center 6 . It may be noted that due to the complexity and insufficient study of gas-dynamic processes, the interest in studying various processes of interaction of a gas jet with a barrier is still strong [31-34]. 


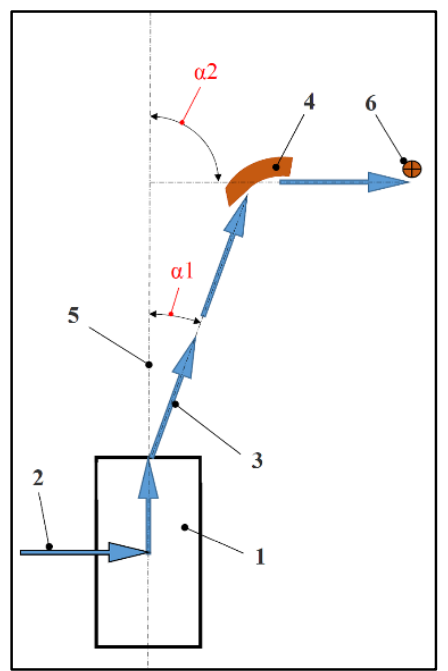

Figure 19. Switchgear diagram - variant No. 2: 1- switchgear; 2- inlet channel; 3- gas jet; 4- deflector; 5- axial line; 6-rotation center.

The presented scheme is the basis of the new turbine design. The developed turbine can be referred to as the group of pulse turbines [35-37]. Jet 3 can periodically interact with a solid wall of deflector 4 . The spatial jet position can periodically change, for example, due to the alternation of variants 2 and 3, according to Figures 19 and 20.

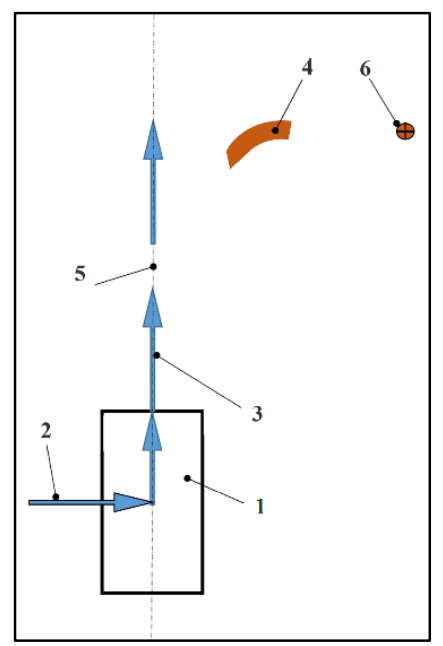

Figure 20. Switchgear diagram - variant No. 3 .

An increase in pressure (Figure 19) or a decrease in pressure (Figure 21), compared with the ambient pressure, can be observed at the contact zone of gas jet 3 with the solid wall of deflector 4 . The reduction of gas (liquid) pressure near the surface of the solid wall may be related to the manifestation of the Coanda effect [38].

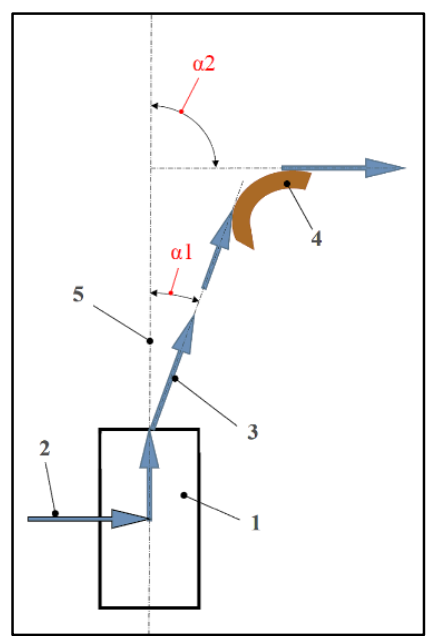

Figure 21. Switchgear diagram - variant No. 4: 1- switchgear; 2- inlet channel; 3- gas jet; 4- deflector; 5- axial line. 
Deflector 4 may be in the form of a tube, as shown in variant No. 5 in Figure 22. The tube itself can be stationary or movable. For example, in the well-known Euler turbine, tubes are included in the rotating rotor.

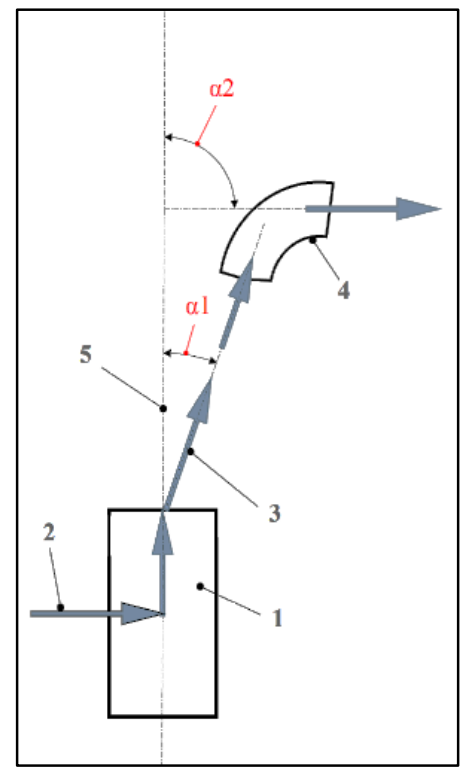

Figure 22. Switchgear diagram - variant No. 5: 1- switchgear; 2- inlet channel; 3- gas jet; 4- piped deflector.

At the stage of exploratory research, about the published information, it was decided to use the FloEFD software package for computer modeling, which allows studying impeller machinery and high-speed jet streams. Thus, computer modeling experts [39] note that comparing the results obtained in the FloEFD, ANSYS Fluent, and RC-VTOL software packages shows satisfactory and good compliance with the experiment in some modes. The implementation of sliding mesh technology for rotation modeling allowed FloEFD to dive into solving complex aerodynamic problems, such as modeling the main helicopter rotor and validate a single rotor blade flow together with the Central Institute of Aerohydrodynamics. In another research, it was concluded that the FloEFD software package could be used to study jet flows of a solid-propellant landing propulsion system, especially at the preliminary design stage, since it reproduces the general structure of the jet and gives satisfactory results for the near flow field with a small investment of time and computational resources [40]. Special diaphragms located at the nozzle outlet can be used to control the flow direction. The design of the adjustable nozzle using a deflector made in the form of a diaphragm is known from U.S. patent No. 3013494 [27]. One of such variants of the switchgear is shown in Figures 23 and 24. In this case, nozzle 1 is equipped with movable diaphragm 2, according to the technical solution presented in the patent [27]. The figure does not show the parts for moving and fixing the diaphragm.

As already noted, a round-hole diaphragm can be called classic. The developed prototype and the patent used a diaphragm with several rectangular holes made on a cylindrical wall [26]. All these diaphragm embodiments operate on the same principles. It was decided to start the research with the classic version, as the simplest variant.

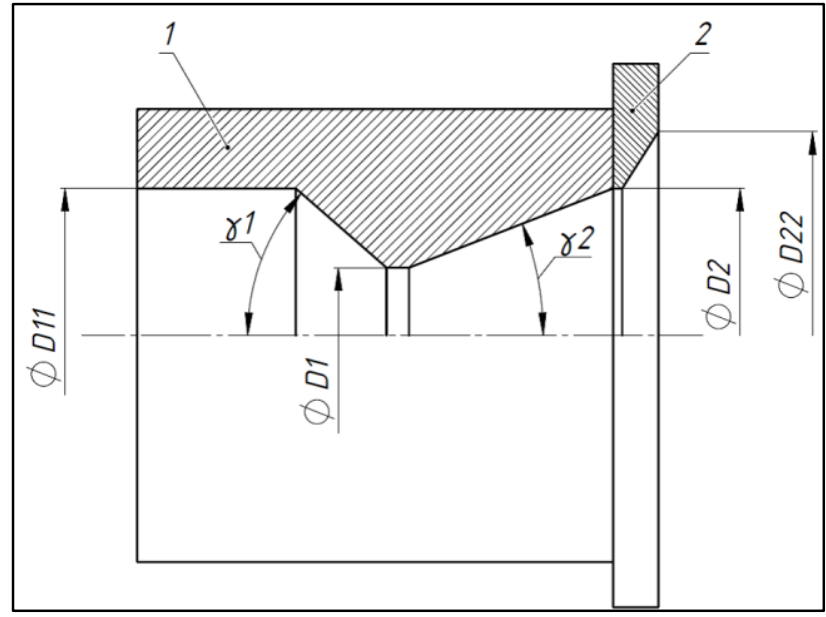

Figure 23. Structural model of a nozzle with a movable diaphragm: 1- nozzle; 2 - the movable diaphragm.

A 3D model of a nozzle with a movable diaphragm is shown in Figure 24. 


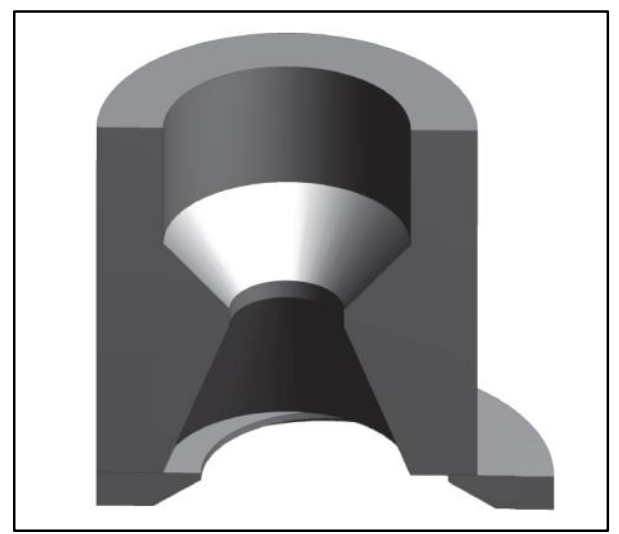

Figure 24. 3D model of a nozzle with a movable diaphragm (view with a radial diaphragm displacement is shown).

Nozzle dimensions: D1 = $8 \mathrm{~mm}$; D1 = $6 \mathrm{~mm}$; D2 = $8.34 \mathrm{~mm}$; D22 = $13 \mathrm{~mm} ; \gamma 1=45$ degrees; $\gamma 2=7$ degrees. A series of calculations were performed to assess the technical capabilities of the known nozzle equipped with a diaphragm. As an example, the conditions of air outflow through the nozzle with the inlet pressure of $1.0 \mathrm{MPa}$ (temperature of 40 ${ }^{\circ} \mathrm{C}$ ) at ambient pressure of $0.1 \mathrm{MPa}$ (temperature of $20{ }^{\circ} \mathrm{C}$ ) are considered. When the nozzle and the diaphragm are arranged concentrically, as shown in Figure 25, the jet at the nozzle outlet extends along the longitudinal nozzle axis. Calculation parameters: total number of cells in the computational grid $=1153165$; computation time $=29298 \mathrm{~s}$; the number of iterations $=1499$.
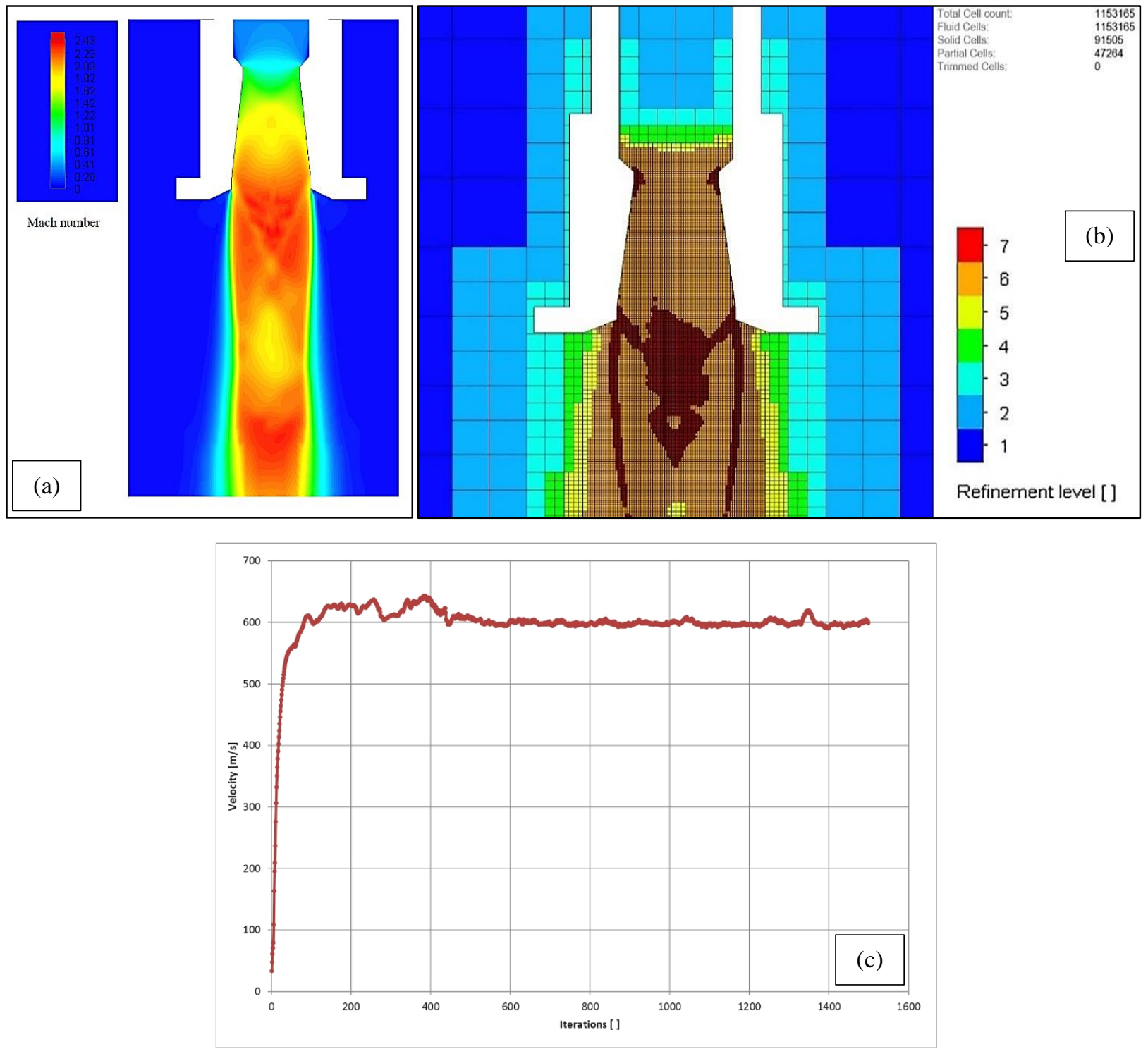

Figure 25. Gas outflow pattern with a concentric arrangement of nozzle and diaphragm: (a) calculation data; (b) A fragment of the adapted computational grid; (c) A fragment of the report on the calculation data convergence. 
When the diaphragm is radially displaced relative to the nozzle, as shown in Figure 26, at the nozzle exit, the jet deviates from the longitudinal axis of the nozzle to a certain angle. Calculation parameters: total number of cells in the computational grid 1137689; computation time 27742 c; iterations 1447.
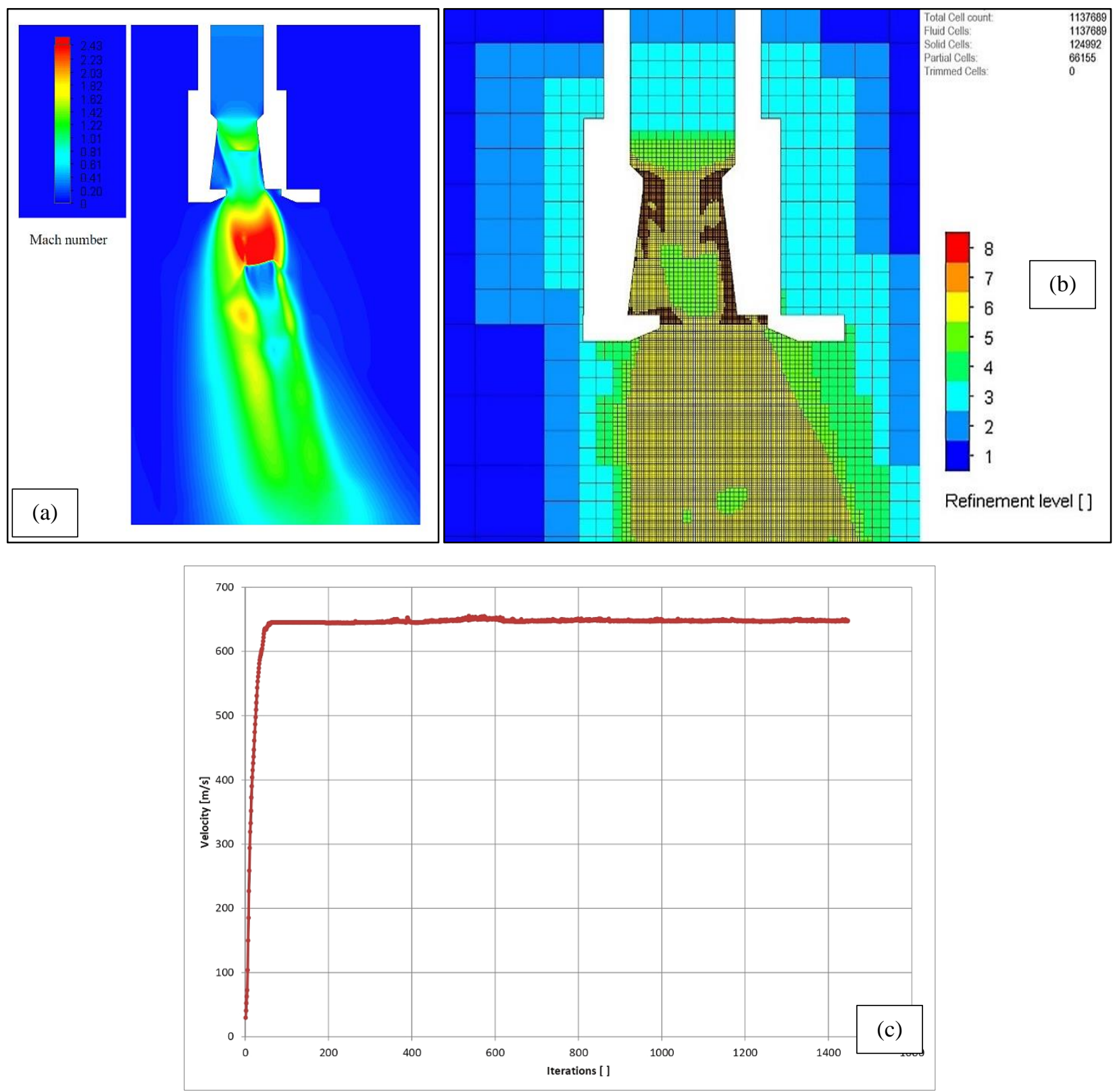

Figure 26. Gas outflow pattern when the diaphragm is radially displaced relative to the nozzle: (a) Calculation data; (b) A fragment of the adapted computational grid; (c) A fragment of the report on the calculation data convergence.

Based on the calculations performed, it can be confirmed that using the known design of the expanding nozzle with a flat diaphragm, the largest jet deflection angle at the nozzle outlet can vary within a narrow range from $+20^{\circ}$ to $-20^{\circ}$. The nozzle design was further modified when the cone angle $\gamma 2$ was increased to $60^{\circ}$ (according to the structural model in Figure 23). A series of calculations were performed to assess the technical capabilities of the modified nozzle equipped with a diaphragm. Calculations were performed for conditions using gaseous and liquid model media, and similar results were obtained in two different media. Different calculation results are presented graphically in Figures 27-29.

Nozzle dimensions: D11 = $12 \mathrm{~mm}$; D1 = $6 \mathrm{~mm}$; D2 = $6 \mathrm{~mm} ; \gamma 1=60$ degrees; $\gamma 2=60$ degrees.

The conditions of water flowing through the nozzle with the inlet pressure of $0.3 \mathrm{MPa}$ at ambient pressure of $0.1 \mathrm{MPa}$ are considered. Angle $\propto$ is marked in the inscription in Figure 18. Calculation parameters: total number of cells in the computational grid 334512; computation time 6974 c; the number of iterations 1189. 

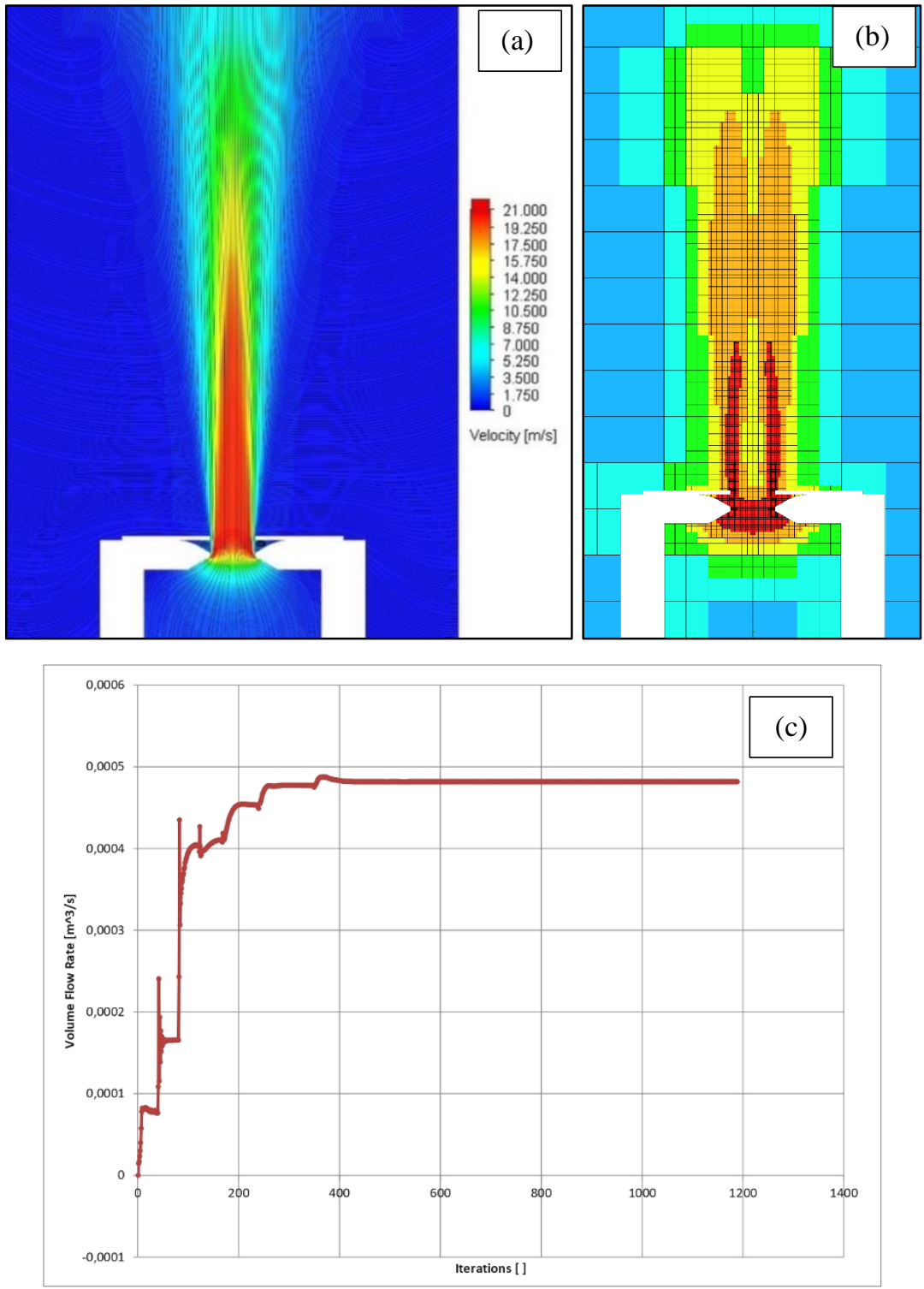

Figure 27. Liquid outflow pattern from a nozzle with a diaphragm for conditions when $\propto=0^{0}$ : (a) Calculation data; (b) A fragment of the adapted computational grid; (c) A fragment of the report on the calculation data convergence.

Calculation parameters: total number of cells in the computational grid 320911; computation time $14404 \mathrm{c}$; the number of iterations 1987.
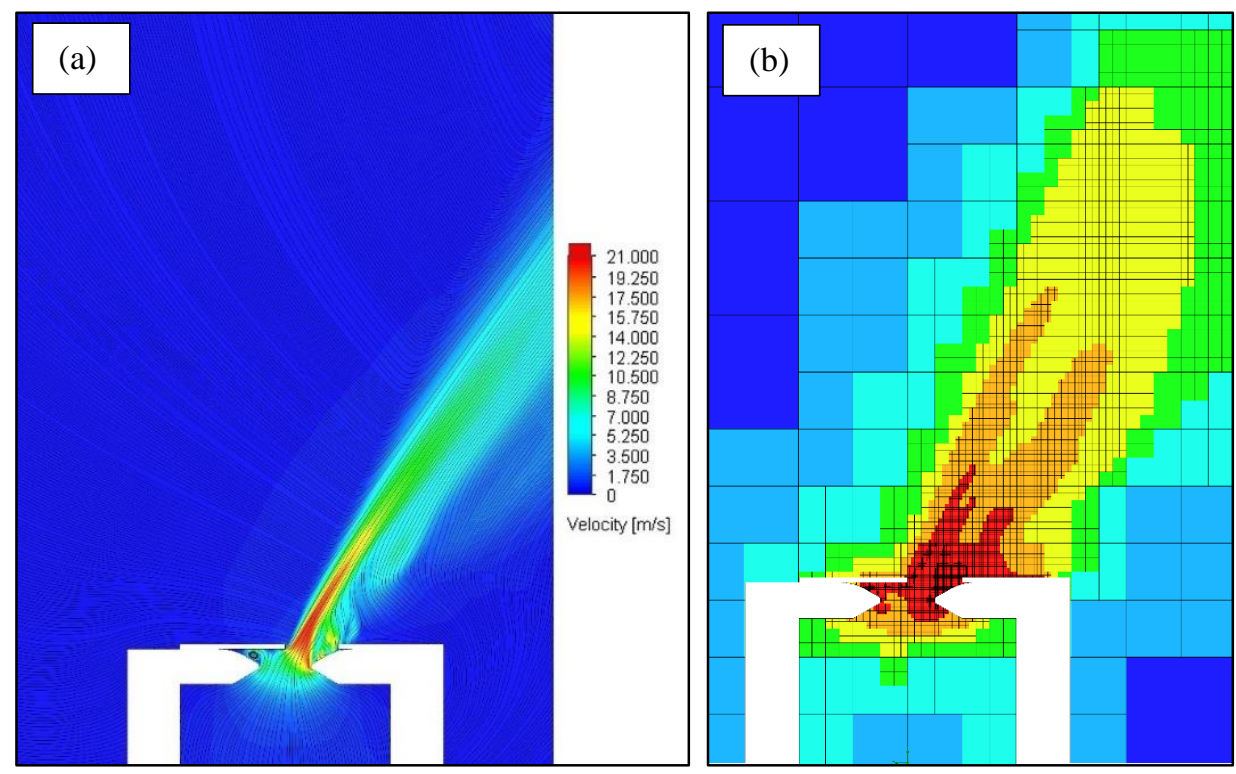


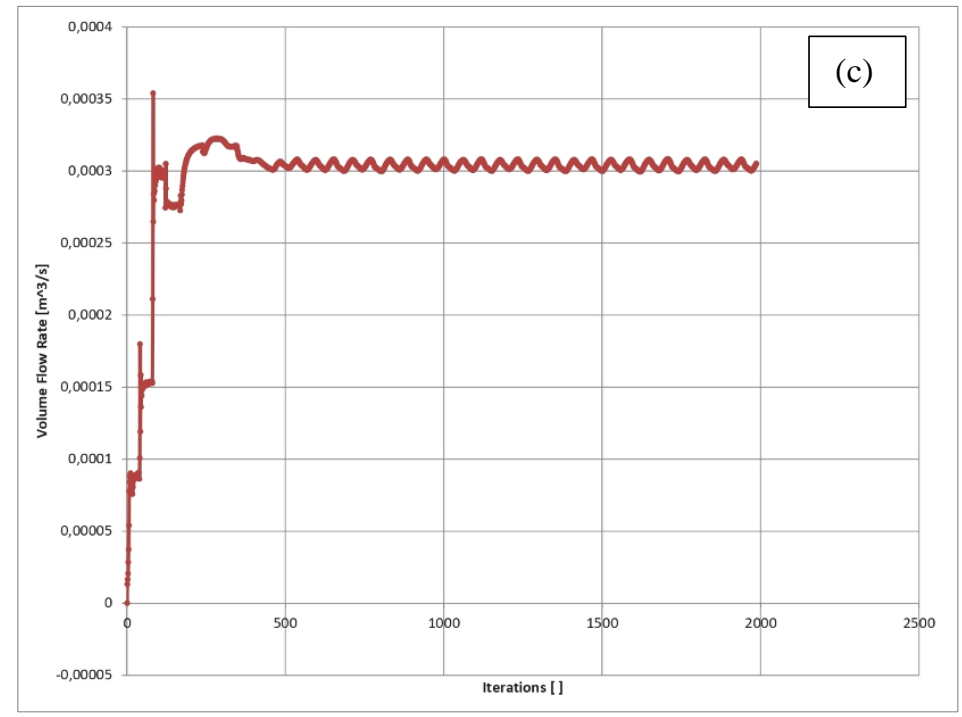

Figure 28. Liquid outflow pattern from a nozzle with a diaphragm for conditions when $\propto$ is more than $0^{\circ}$ but less than $90^{\circ}$ : (a) Calculation data; (b) A fragment of the adapted computational grid; (c) A fragment of the report on the calculation data convergence.

Calculation parameters: total number of cells in the computational grid 322292; computation time $2772 \mathrm{c}$; the number of iterations 610 .
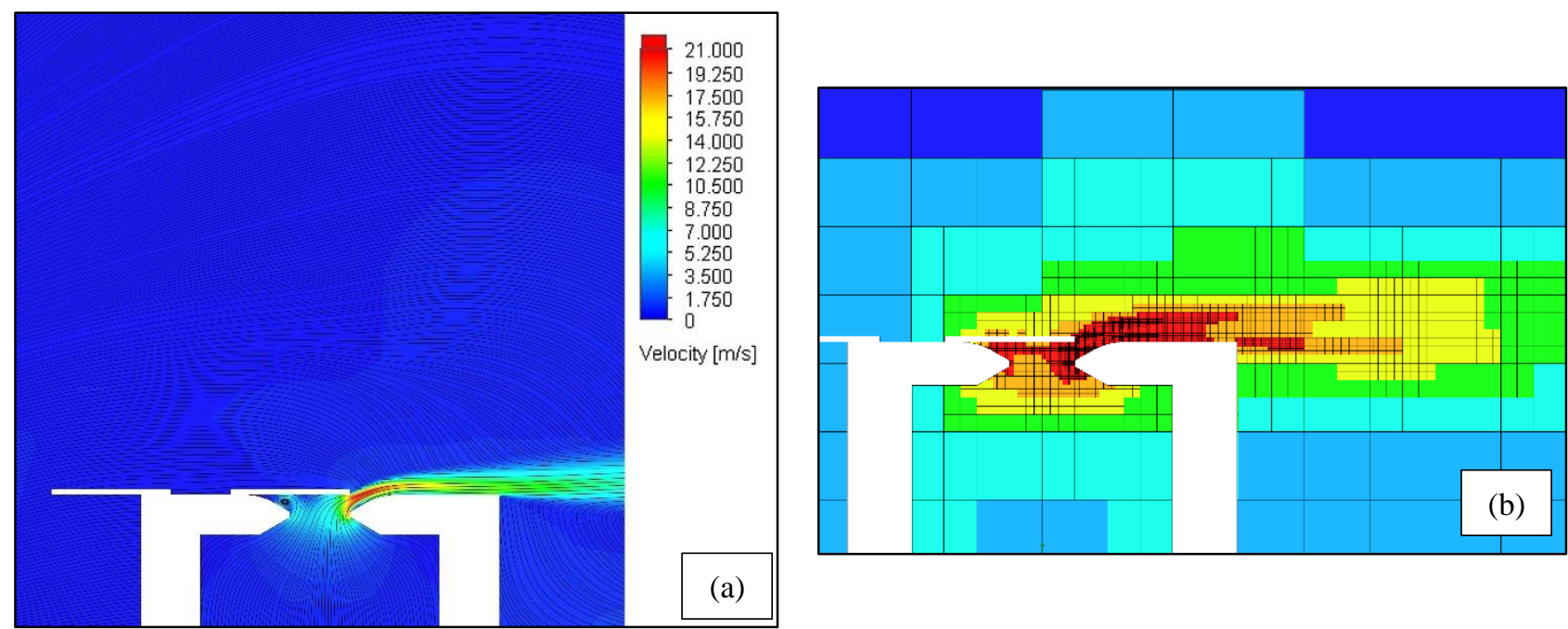

(a)

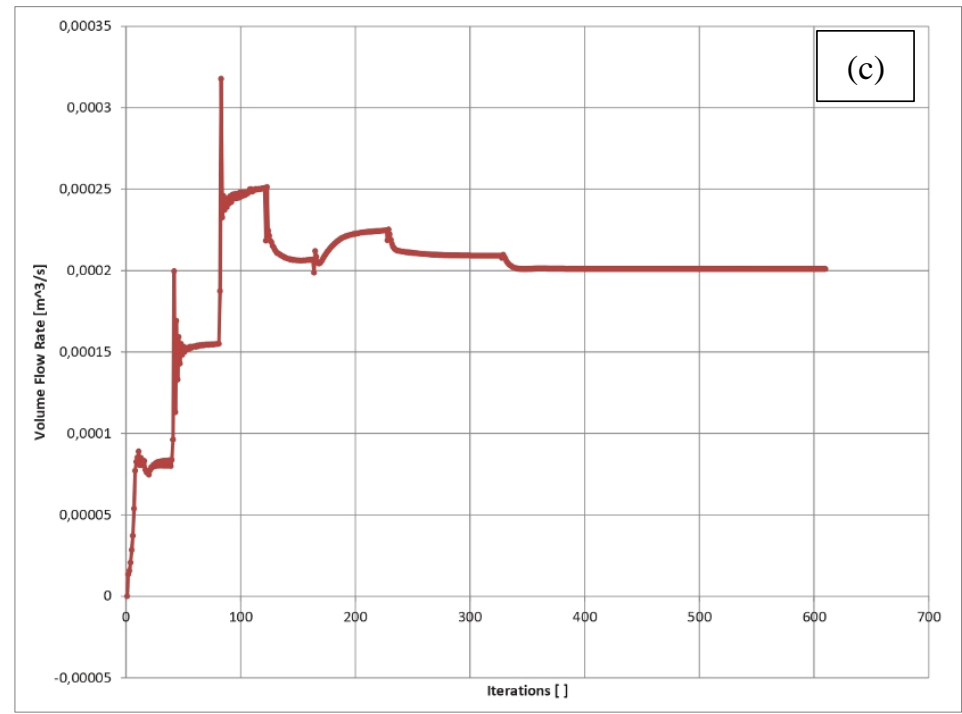

Figure 29. Liquid outflow pattern from a nozzle with a diaphragm for conditions when $\propto=90^{\circ}$ : a) calculation data; b) a fragment of the adapted computational grid; c) a fragment of the report on the calculation data convergence. 
The computer simulation results (Figures 27 to 29) showed the possibility of the velocity vector deflection (at the nozzle outlet) at an angle ranging from $+90^{\circ}$ to $-90^{\circ}$ within the geometric hemisphere, and the velocity vector was controlled using a flat diaphragm. Earlier, similar results of the physical experiment [1, 23, 24] performed under laboratory conditions using a gaseous and a liquid model medium were obtained and published. Summarizing the results of the performed numerical experiments and the results of previously performed physical experiments, we can make an intermediate conclusion about the scientific novelty of the research as a whole: in the course of physical and numerical experiments, for the first time, the extreme conditions for the outflow of liquid and gas through a nozzle equipped with a velocity vector control system were considered, in the control range for the velocity vector deviation angle from $+180^{\circ}$ to $-180^{\circ}$ within the geometric sphere.

Using computer simulation, by calculation, we have confirmed the possibility of deflecting the jet by 90 degrees with a control system equipped with a movable diaphragm. This result complies with the result of the physical experiment described earlier in our publications [1,23]. A previous paper [24] also noted that mesh turbines could be developed based on the Euler turbine. In this case, the curved tube is used as a mixing chamber for the ejector. A set of such mixing chambers is placed in the turbine rotor, and the ejector nozzle remains fixed. The pulse mode of gas (or liquid) flow is realized in the rotor flow channels with such a design. In this regard, the scheme of the jet system (Figure 22) can be considered a basis for developing special turbines (or jet systems) of this type. In inflow channels of such systems, two working processes are realized simultaneously: turbine and ejection working processes. As a result, we can talk about the prospect of developing hybrid turbomachines. Analysis of available jet control systems showed that the example with a movable diaphragm is only one of many ways to control gas flows at the nozzle outlet, so it is advisable to continue research in this direction.

\section{5-1- Implication and Explanation of Findings}

Based on the computer simulation results, it is possible to partially confirm or repeat the conclusion of specialists and authors of [40]: the FloEFD software package used in the simulation reproduces the general flow structure and gives satisfactory results for the near flow field with low time and computing resource consumption. This paper considered only one particular case using a nozzle in which the cone angle $\gamma 2$ was increased to $60^{\circ}$ (according to the diagram in Figure 23). That helped to radically expand the control range for the deflection angle velocity vector. It seems that there are many other methods and possibilities to extend the control range for most jet parameters, including such a parameter as the deflection angle of the velocity vector (thrust vector). Therefore, there is a great prospect for improving the technical performance of such control systems, and scientific and patent research is advisable to continue in this direction.

In physical and numerical experiments, it was observed that even an axisymmetric circular jet significantly changes its shape in the case of radial displacement of the regulating diaphragm (Figures 27-29). In this regard, an adjustable nozzle of this type can be called an unconventional nozzle. For this type of nozzle, computer simulation and special calculations are required to determine the Coriolis coefficient $\propto_{k}$ and the Boussinesq coefficient $b_{k}$ values. These coefficients will further be included in the momentum equation (Euler equation) when calculating jet devices of various designs, as part of the revision of the published calculation methodology [41] for ejector systems:

$$
\begin{aligned}
& \propto_{k}=F_{k}^{-1} \int_{F_{k}} \bar{v}^{3} d F, \\
& b_{k}=F_{k}^{-1} \int_{F_{k}} \bar{v}^{2} d F,
\end{aligned}
$$

where $\bar{v}=\frac{v}{v_{s}}$ : relative flow velocity; $v_{s}$ : average flow velocity; $F_{k}$ : an area of flow section; F: an area of channel section. In this case, the results of each such calculation will depend on the value of radial displacement of the regulating diaphragm. The parameters $F_{k}$ and $\mathrm{F}$ will change their values depending on the location of the regulating diaphragm. Detailed study of effects with jet deformation is important for considering heat exchange processes and processes during jet interaction with solid walls of different types. Studying the temperature distribution pattern along the length of this deformed jet (especially in the infrared spectrum) is interesting both from a scientific and practical point of view. Preliminary calculations have shown that the mesh turbine can have sufficiently high-efficiency indicators, which opens up the prospects for wide practical use of such equipment.

Computer simulation and modern computational methods help to accelerate research work. However, as specialists note, a model of any level of complexity cannot claim to describe all properties of real turbulent flows adequately, and the problem of calculating turbulent flows will remain an important and topical problem of theoretical and computational fluid mechanics for a long time [31, 42]. In this regard, an important part of the research work is to conduct physical experiments in laboratory bench conditions, with the corresponding development of prototypes, models, and layouts. In 
design and engineering work, the term prototype usually refers to a working model created to demonstrate and confirm the product's performance under development. The technology of quick prototyping provides a rapid realization of the basic functionality of the future product. Currently, rapid prototyping has become possible for a wide range of researchers and specialists through various additive technologies, including 3D printers.

\section{5-2- Strengths and Limitations}

Figures 30-32 show photos of the developed prototypes manufactured using 3D printers. Also, the pneumatic and hydraulic tests' results confirmed the mesh turbine's suitability for its operation in conditions of changing the working medium density in a wide range from 1 to $1.000 \mathrm{~kg}$ per cubic meter. These results of pneumatic and hydraulic tests were described in $[1,23,24]$.

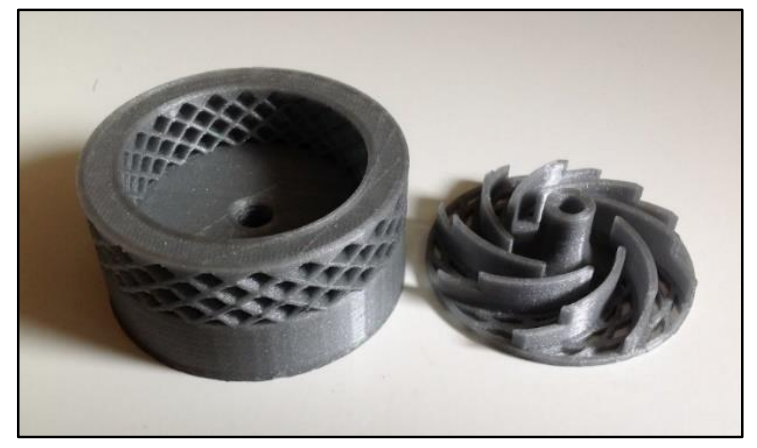

Figure 30. Micromodels of a mesh turbine (left) and a vane pump wheel (right).

In the figures below, arrows schematically show the working fluid flow $Q_{0}$ and the pumped medium flow $Q_{1}$. The turbopump casing is not shown in these figures.

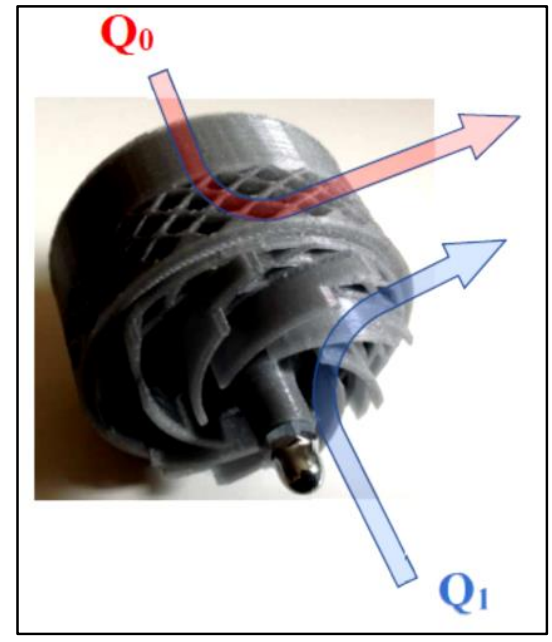

Figure 31. Rotor micromodel for a turbo-pump with radial mesh turbine.

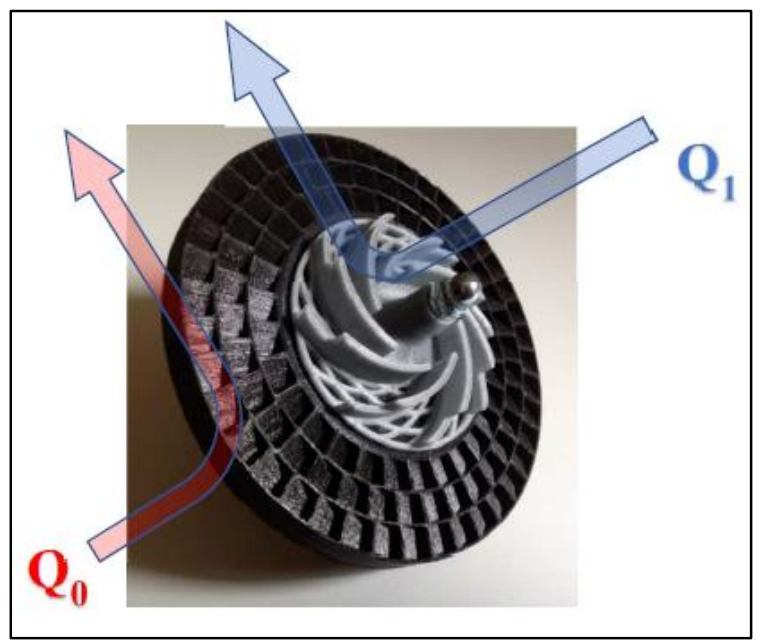

Figure 32. Micromodel of an experimental rotor with an axial mesh turbine. 
In a further study of gas-dynamic processes in channels with a mesh structure, it will be necessary to consider choked flow conditions. One of the main features of the medium compressibility impact on its interaction with the permeable body is the choked flow conditions. In this case, the parameters on the windward side of the permeable body boundary cease to depend on the conditions on its leeward side. Then, any medium leakage laws linking the pressure drop at the permeable boundary with the parameters of the oncoming flow become unsuitable in principle, and there is a need to develop new experimental ways of determining the boundary relations [43-45].

It is known that the use of features of jet streams flowing from unconventional nozzles makes it possible to meet a variety of requirements arising in the design of technological processes [6, 46]. The use of a nozzle with a nonaxisymmetric outlet section allows for a significant increase or decrease in the force impact on a barrier [46]. Many researchers note that the three-dimensional gas-dynamic flow structure in nozzles with an unconventional shape of the critical section has not been sufficiently investigated [40]. The round, square, plane, and radial air (annular) jets have been investigated extensively [47]. Scientific publications present the results of research and development of new nozzle configurations of rocket engines, mainly annular (with internal and external flow expansion) for dense configurations of advanced missile stages and spacecraft [48]. The results of these studies have shown that known gas-dynamic methods of thrust vector control can be effectively used in expansion-deflection nozzles. At the same time, the existence of a relatively small-sized central body in this nozzle makes it possible to use new mechanical methods based on transverse displacement or oscillation of the central body. To clarify technical issues, Figures 33-36 show some materials obtained during the demonstration experiments. Some of these materials are supposed to be used in the educational process to familiarize students with the features of hybrid workflows in the theory of turbomachines. In one of the demonstration experiments, an aircraft model fan with a diameter of $55 \mathrm{~mm}$ was used as an impeller, Figure 33.

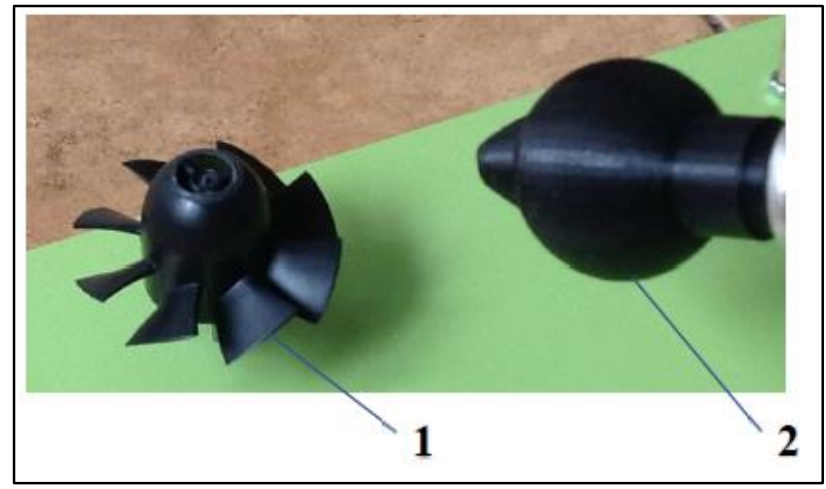

Figure 33. The main elements of the experimental system are in a static state: 1- impeller; 2- nozzle.

In Figure 34, arrows schematically show the working fluid flow $Q_{0}$ and the pumped medium flow $Q_{1}$. In this case, air flows are shown.

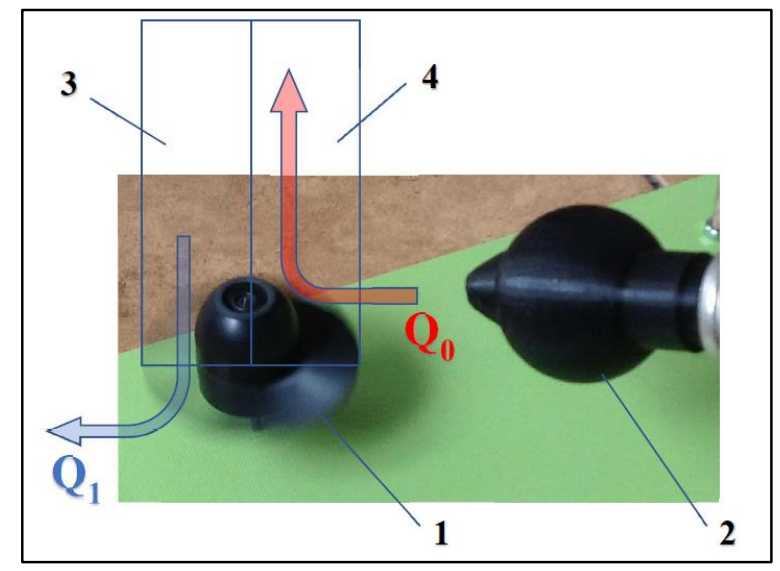

Figure 34. The main elements of the experimental system in a dynamic state: 1- impeller; 2- nozzle; 3- fan operation zone; 4- turbine operation zone

When air is supplied through nozzle 2 in impeller 1, two workflows run simultaneously. The first process is the turbine workflow. In the diagram, the part of the impeller located to the right of the rotation axis participates in implementing the turbine workflow. The airflow leaving nozzle 2 exerts a force effect on the blades of impeller 1 . The kinetic energy of the working fluid flow $Q_{0}$ is converted into mechanical energy, which is transferred to the impeller, and the impeller is involved in rotational motion. The operation area for the turbine process is schematically indicated in the figure as zone 4. 
The second process is the fan workflow. In the diagram, the part of the impeller located to the left of the rotation axis participates in implementing the second workflow. The impeller blades exert a force effect on the pumped medium and create (form) the flow $Q_{1}$. The ambient air is used as the pumped medium. The operation area for the fan workflow is schematically indicated in the figure as zone 3. Each blade of rotating impeller 1 passes through zone 3 and zone 4 periodically. In this case, a pulsed gas flow mode is implemented in the channels between the impeller blades. If the air supply to nozzle 2 is stopped, zone 4 will disappear. Moreover, all blades of impeller 1 will participate in the fan workflow implementation. When impeller 1 rotates, the stock of kinetic energy may be enough for impeller 1 to take off vertically upward, overcoming the action of gravity, as shown in Figure 35. This figure shows individual frames from the video recording, and the time in seconds is indicated under each frame, according to the timer readings on the camcorder.

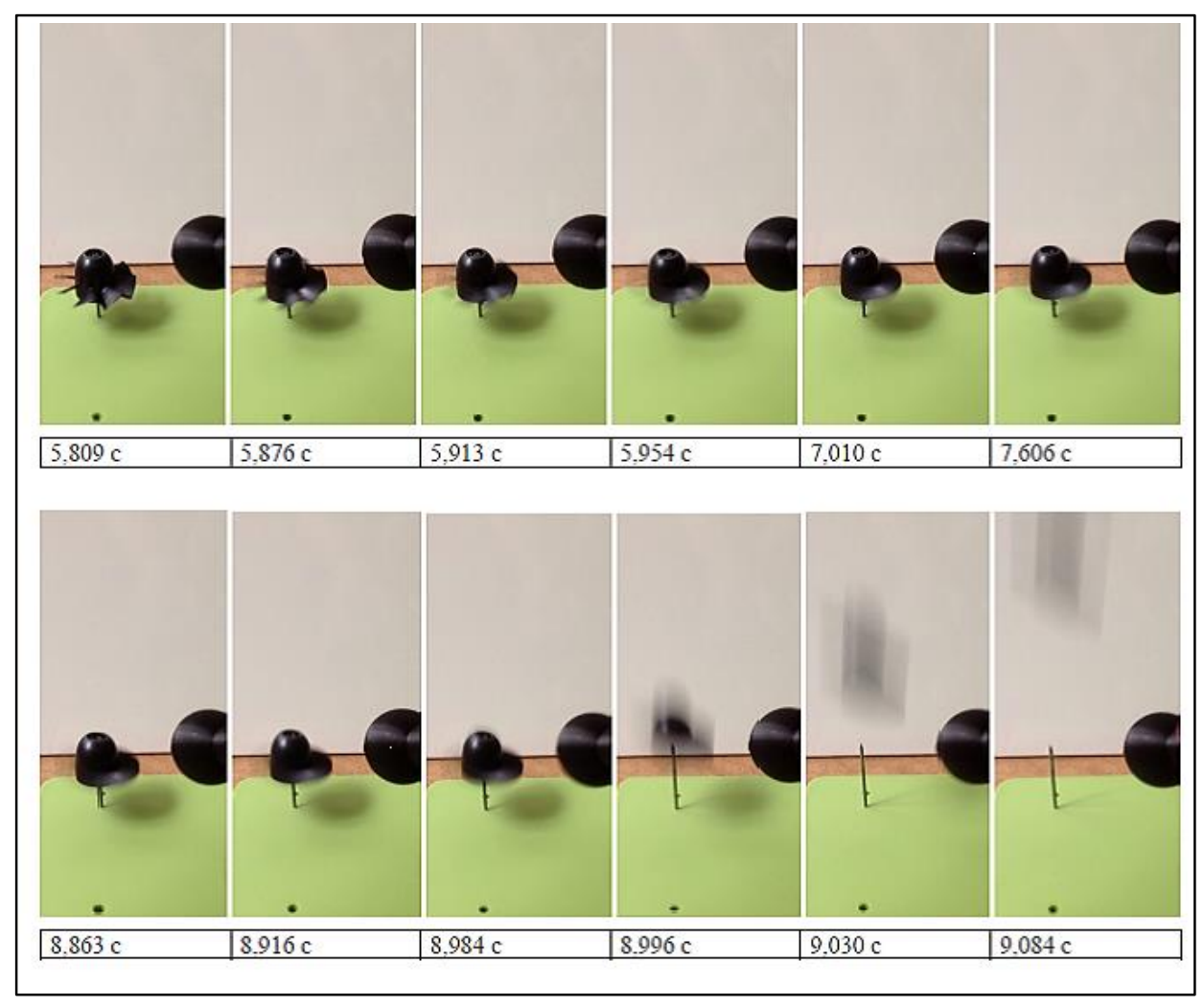

Figure 35. A set of frames from the video recording of the demonstration experiment.

Similar demonstration experiments were also carried out using the prototype mesh turbines with a mesh rotor diameter of up to $120 \mathrm{~mm}$, Figure 36.

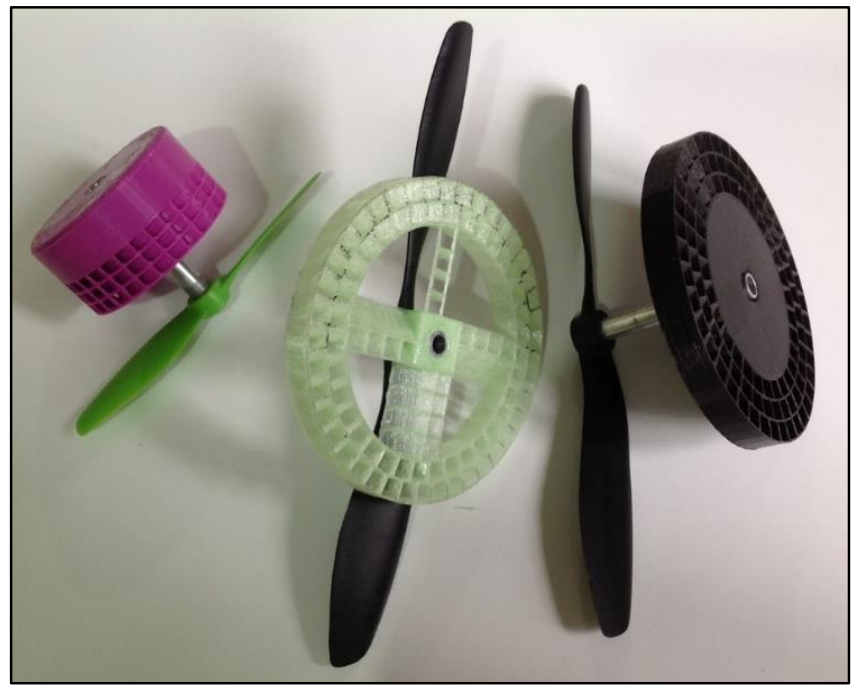

Figure 36. Samples of mesh turbines with propeller screws were prepared for demonstration experiments.

Earlier, it was already noted in [41] that the turbine workflow could manifest itself in the channels of impeller pumps during some operating modes of the pumping unit. The expediency of scientific research of the relationship between the 
pumping and the turbine workflows in the channels of one impeller was emphasized. The research development will relate to the study of the peculiarities of gas-dynamic and hydrodynamic processes in the pulsed flow of gases and liquids through channels with a mesh structure. Because of the variety of practical tasks and the significant amount of research work involved, the features of nozzles and ejectors with unconventional geometry can be studied in a separate scientific work.

\section{5-3- Summary}

The development of research will also relate to the study of acoustic characteristics of mesh turbines and nozzles. Modern calculation methods make it possible to solve such problems. In particular, the results of experimental studies of the acoustic characteristics of subsonic turbulent jets flowing from rectangular nozzles are known, and they show that the directivity of sound radiation and noise spectra in the far acoustic field of such jets are close to the corresponding characteristics of a jet flowing from a round nozzle [49]. The research results are mainly focused on the development of multimode mesh turbomachinery operating under difficult conditions. The work results can be used in various industries, including energy, transport, and robotics. In robotics, there is potential for the development of miniature turbomachines and jet engines with ejector augmenters.

\section{6- Conclusions}

\section{6-1- Scientific Novelty}

During numerical experiments, the extreme conditions of liquid and gas outflow through a nozzle equipped with a velocity vector control system, in the control range for the velocity vector deflection angle from $+90^{\circ}$ to $-90^{\circ}$ within a geometric hemisphere, were considered for the first time. A prototype of a new mesh turbine equipped with a rotary cylindrical diaphragm (movable control sleeve) to control the velocity vector at the nozzle outlet has been developed and patented. The computer simulation results confirmed the possibility of developing a jet system that allows the deflection angle of the velocity vector (thrust vector) to be adjusted in the range from $+90^{\circ}$ to $-90^{\circ}$ within the geometric hemisphere for the conditions of one-outlet nozzle application. It was also shown that when using a two-output nozzle, there are possibilities for adjusting the deflection angle of the velocity vector (thrust vector) in the range from $+180^{\circ}$ to $-180^{\circ}$ within the geometric sphere. These calculated data are presented in addition to previously published results of physical laboratory experiments.

\section{6-2- Theoretical Contributions}

For the conditions of the other ejector work process in the rotor channels, a new direction for the theory of impeller machines has been proposed. There is a plan to develop underlying mathematical models to describe the ejector work process, considering the application of several mixing chambers with channels that form a mesh structure. Preliminary results of numerical experiments show the feasibility of creating a new theory.

\section{6-3- Practical Importance}

The results of the ongoing research can be used to improve the energy efficiency of various technological systems, including systems for extracting and processing hydrocarbons. Some research results can be applied in robotic engineering and the development of small unmanned aerial vehicles.

\section{6-4- Limitations and Future Studies}

At this stage, calculated studies are being carried out using either a gas or a liquid medium. Physical experiments have shown the possibility of working with gas-liquid mixtures, and subsequent work will focus on studying gas-liquid flows in channels with a mesh structure. As such, continued research will involve the study of turbomachinery and ejectors with a mesh structure in the flow channels.

\section{7- Declarations}

\section{7-1-Author Contributions}

Conceptualization, Y.A.S. and M.A.M.; Data curation, V.V.V.; Formal analysis, Y.A.S.; Funding acquisition, M.A.M.; Investigation, M.A.M.; Methodology, I.V.G.; Project administration, M.A.M.; Resources, I.V.G.; Software, V.V.V.; Supervision, Y.A.S.; Validation, K.A.T., M.A.F. and N.N.B.; Visualization, N.N.B.; Writing - original draft, K.A.T.; Writing - review \& editing, M.A.F. All authors have read and agreed to the published version of the manuscript.

\section{7-2-Data Availability Statement}

The data presented in this study are available in article. 


\section{7-3- Funding}

The work has been performed with the financial support of the Ministry of Education and Science of the Russian Federation within the framework of the state contract in the area of scientific activities, topic number FSZE-2020-0006.

\section{7-4- Conflicts of Interest}

The authors declare that there is no conflict of interests regarding the publication of this manuscript. In addition, the ethical issues, including plagiarism, informed consent, misconduct, data fabrication and/or falsification, double publication and/or submission, and redundancies have been completely observed by the authors.

\section{8- References}

[1] Sazonov, Yu. A., M. A. Mokhov, Kh. A. Tumanyan, M. A. Frankov, and N. N. Balaka. "Prototyping mesh turbine with the jet control system.” Periódico Tchê Química 17, no. 36, (2020): 1160-1175.

[2] Konovalova, N.E., and T.M. Pritulo. "Experimental and computational study of the flow around a lattice wing model of honeycomb structure." Air Fleet Technique 4, no. 705, (2011): 25-28. Available online www.tsagi.ru/institute/publications/ machinery_fleet/archive_annotations/2011/TBФ\%20№4-2011.pdf (accessed on May 2021).

[3] Drozdov, S.M. "Method of determining the full-scale aerodynamic performance of an airplane with lattice wings based on the results of its model wind-channel tests.” TsAGI Science Journal XXXIII, no. 3-4, (2002): 18-29.

[4] Kostyukov, A.V.; Nadarejshvili, G.G.; Karpukhin, K.E.; Tuktakiev, G.S.; Azarov, K.O. High-Temperature Rotating Disk Regenerative Heater of the Working Body of the Power Plant. RF Patent 2,744,926. Available online: https://patenton.ru/patent/RU2744926C1.pdf (accessed on 10 April 2021).

[5] Orman, Ł. J. (2020). Aspects of Complexity of Metal-Fibrous Microstructure for the Construction of High-Performance Heat Exchangers: Thermal Properties. Aviation, 24(3), 99-104. doi:10.3846/aviation.2020.12086

[6] Giuliani, F., M. Stütz, N. Paulitsch, and L. Andracher. "Forcing pulsations by means of a siren for gas turbine applications." International Journal of Turbomachinery, Propulsion and Power 5, no. 2, (May 13, 2020): 9. doi:10.3390/ijtpp5020009.

[7] Kalachev, Vladimir Viktorovich. “Jet Pumps. Theory, Calculation, and Design” (2017).

[8] Bogdanov, Vasiliy Ivanovich, Aleksey Konstantinovich Dormidontov, and Vladimir Vassilievich Yakovlev. "RF Utility Model Patent No. 202,545. Ejector Augmenter. Bulletin № 6” (February 24, 2021).

[9] Berezhnoy, A.S. "Improving the Performance of Jet Reaction Pneumatic Unit Based on the Model Update of the Work Process" (2014). Available online: https://essuir.sumdu.edu.ua/bitstream-download/123456789/36816/6/dis_Berezhnoi.pdf;jsessionid= 8AD8CAEA6B37A33DC7BF64A1E74C99BF (accessed on May 2021).

[10] Sazonov, Yuriy Apollonievich, Mikhail Albertovich Mokhov, K.A. Tumanyan, Mikhail Alexandrovich Frankov, and Victor Gennadievich Timoshenko. "RF Utility Model Patent No. 192,513. Motor. Bulletin № 26.” (September 18, 2019).

[11] Sarwar Abbasi, W., S. Ul Islam, L.Faiz, and H. Rahman. "Numerical investigation of transitions in flow states and variation in aerodynamic forces for flow around square cylinders arranged inline." Chinese Journal of Aeronautics 31, no. 11, (November 2018): 2111-2123.doi:10.1016/j.cja.2018.08.020.

[12] Shin, Hyung Ki, Jun Hyun Cho, Young Jin Baik, Gil Bong Lee, BeomJoomLee, ChuiWooRoh, HoSangRa, and JongJaeCho. "United States Patent 11028724. Partial Admission Operation Turbine Apparatus for Improving Efficiency of Continuous Partial Admission Operation and Method for Operating Turbine Apparatus Using Same" (June 8, 2021).

[13] Silvestri, George J. Jr. "United States Patent 4780057. Partial Arc Steam Turbine.” (October 25, 1988).

[14] Matsumoto, Hiroshi, Yoshiyuki Nakano, Toshihiko Higashi, and Akihiro Yasumoto. "United States Patent 4120159. Steam Turbine Control System and Method of Controlling the Ratio of Steam Flow between under Full-Arc Admission Mode and under Partial-Arc Admission Mode" (October 17, 1978).

[15] Ahmad, K., Y. Baig, H. Rahman, and H. Junaid Hasham. "Progressive failure analysis of helicopter rotor blade under aeroelastic loading." Aviation 24, no. 1, (April 23, 2020): 33-41. doi:10.3846/aviation.2020.12184.

[16] Ignatkin, Y., P. Makeev, S. Konstantinov, and A. Shomov. "Modelling the helicopter rotor aerodynamics at forward flight with free wake model and Urans method." Aviation 24, no. 4, (November 9, 2020): 149-156. doi:10.3846/aviation.2020.12714.

[17] Ding, X., P. Guo, K. Xu, and Y. Yu. "A review of aerial manipulation of small-scale rotorcraft unmanned robotic systems." Chinese Journal of Aeronautics 32, no. 1, (January 2019): 200-214. doi:10.1016/j.cja.2018.05.012.

[18] Parancheerivilakkathil, M.S., R.M. Ajaj, and K.A. Khan. “A compliant polymorphing wing for small UAVs.” Chinese Journal of Aeronautics 33, no. 10, (October 2020): 2575-2588. doi:10.1016/j.cja.2020.03.027.

[19] Neigapula, S.N.V., S.P. Maddula, and V.B. Nukala. "A study of high lift aerodynamic devices on commercial aircraft." Aviation 24, no. 3, (September 23, 2020): 123-136. doi:10.3846/aviation.2020.12815. 
[20] Zhang, J., L. Sun, X. Qu, and L. Wang. “Time-varying linear control for tiltrotor aircraft.” Chinese Journal of Aeronautics 31 , no. 4, (April 2018): 632-642. doi:10.1016/j.cja.2018.01.025.

[21] Sun, Z., B. Wang, X. Zheng, T. Kawakubo, H. Tamaki, and R. Numakura. "Effect of bent inlet pipe on the flow instability behavior of centrifugal compressors." Chinese Journal of Aeronautics 33, no. 8, (August 2020): $2099-2109$. doi:10.1016/j.cja.2020.02.013.

[22] Sieverding, C., and M. Manna. “A review on turbine trailing edge flow.” International Journal of Turbomachinery, Propulsion and Power 5, no. 2, (May 20, 2020): 1-55. doi:10.3390/ijtpp5020010.

[23] Sazonov, Yu. A., Mikhail Albertovich Mokhov, I.A. Gryaznova, V.V. Voronova, K.A. Tumanyan, M.A. Frankov, V.A. Moon, and N.N. Balaka."Prototype development of intelligent mesh turbine using the ejector control system." Gas Industry 11, (November 2020):52-59. Available online: https://neftegas.info/gasindustry/-11-2020/razrabotka-prototipa-intellektualnoysetchatoy-turbiny-s-ispolzovaniem-ezhektornoy-sistemy-upravleni/ (accessed on April 2021).

[24] Sazonov, Yuri Appolonievich, Mikhail Albertovich Mokhov, Inna Vladimirovna Gryaznova, Victoria Vasilievna Voronova, Vladimir Valentinovich Mulenko, Khoren Arturovich Tumanyan, Mikhail Alexandrovich Frankov, and Nikolay Nikolaevich Balaka. "Prototyping and Study of Mesh Turbomachinery Based on the Euler Turbine." Energies 14, no. 17 (August 26, 2021): 5292. doi:10.3390/en14175292.

[25] Scott, J. "Missile Control Systems." Aerospaceweb (January 11, 2004). Available online: http://www.aerospaceweb.org/ question/weapons/q0158.shtml (accessed on August 2021).

[26] Sazonov, YuriyApollonievich, MikhailAlbertovichMokhov, KhorenArturovichTumanyan, VictoriaVasilyevnaVoronova, and MikhailAlexandrovichFrankov.“RF Patent No. 203833. Motor. Bulletin № 12” (April 24, 2021).

[27] Chanut, Pierre Louis Jean. “US Patent № 3,013,494. Guided Missile.” (December 19, 1961). Available online: http://www.freepatentsonline.com/3013494.pdf (accessed on August 2021).

[28] Zhao, K., M. Ming, F. Li, Y. Lu, T. Zhou, K. Wang, and N. Meng. "Experimental study on plasma jet deflection and energy extraction with MHD control." Chinese Journal of Aeronautics 33, no. 6, (June 2020): 1602-1610. doi:10.1016/j.cja.2020.01.003.

[29] Abugov, Daniil Isaakovich, and V.M. Bobylev. "Theory and Calculation of Solid Rocket Motors. Textbook for Mechanical Engineering Universities" (1987).

[30] Bailey, John M. “US Patent № 4355949. Control System and Nozzle for Impulse Turbines.” (October 26, 1982). Available online: https://www.freepatentsonline.com/4355949.pdf (accessed on April 2021).

[31] Glaznev, Vladimir Nikolaevich,Valeriy Ivanovich Zapryagaev, Vladimir Nikolaevich Uskov, Natalya Mikhailovna Terekhova, V.K. Erofeev, V.V. Grigoryev, A.O. Kozhemyakin, V.A. Kotenok, and Alexandr Vladimirovich Omelchenko. "Jet and Nonstationary Flows in Gas Dynamics" (2000). Available online: https://www.rfbr.ru/rffi/ru/books/o_25930 (accessed on August 2021).

[32] Mordasov, M.M., A.P. Savenkov, and K.E. Chechetov. "On the clarification of the calculated dependences of the turbulent gas jet force action". Journal of Technical Physics 85, no. 10, (March 5, 2015): 141-144.

[33] Pakhomov, M.A., and V.I. Terekhov."Intensification of turbulent exchange in the interaction of fog-like axisymmetric impact jet with a barrier.” Applied Mechanics and Technical Physics 52 no. 1, (2011): 119-131.

[34] Sadin, D. V., S. D. Lyubarskii, and Yu. A. Gravchenko. "Features of an Underexpanded Pulsed Impact Gas-Dispersed Jet with a High Particle Concentration.” Technical Physics 62, no. 1 (January 2017): 18-23. doi:10.1134/s1063784217010194.

[35] Tarasov, Vladislav Nicolaevich. "Development of Rational Methods of Designing Partial Pulse Turbines" (August 6, 2009). Available online: https://www.dissercat.com/content/razrabotka-ratsionalnykh-metodov-proektirovaniya-partsialnoimpulsnykh-turbin (accessed on May 2021).

[36] Dovgyallo, A.I., and A.A. Shimanov. "The possibility of using a bidirectional impulse turbine in a thermoacoustic engine." Bulletin of Samara State Aerospace University 14, no. 1, (2015): 132-138. doi:10.18287/1998-6629-2015-14-1-132-138.

[37] Konchakov, Evgeniy Ivanovich. "Improvement of Marine Partial Turbomachinery on Small-Sized Models" (October 31, 2001). Available online: https://www.dissercat.com/content/sovershenstvovanie-sudovykh-partsialnykh-turbomashin-na-malykhmodelyakh (accessed on April 2021).

[38] Ilyina, T.E., and N.V. Prodan. "Designing a jet control system element for gas bearing." Scientific and Technical Journal of Information Technologies, Mechanics and Optics 15, no. 5, (2015): 921-929.

[39] Kritskiy, B.S., M.S. Makhnev, R.M. Mirgazov, P.N. Subbotina, and T.V. Trebunskikh. "Aerodynamic characteristics calculation on single rotor blade using FloEFD, ANSYS Fluent and RC-VTOL.” Civil Aviation High Technologies 223, (2016): 77-83.

[40] Antipova, M.S., A.A. Dyadkin, V.I. Zapryagaev, and A.N. Krylov. "Computer simulation of cold supersonic jet outflow from the conical nozzle using FLOEFD software package.” Space Engineering and Technology 1, no. 12, (2016): 5-11. 
[41] Sazonov, Yu. A. "Fundamentals of Calculating and Designing Pumping and Ejector Installations" (2012).

[42] Garbaruk, Andrey Victorovich. "Numerical Modeling and Stability Analysis of Near-Wall Turbulent Flows" (2020). Available online: https://elib.spbstu.ru/dl/2/r20-24.pdf/view (accessed on May 2021).

[43] Sinyavin, Alexey Alexandrovich. "Calculation and Experimental Study of the Interaction of Gas Flows with Permeable Boundaries" (2010).

[44] Xue, Y., L. Wang, S. and Fu, Song."Detached-eddy simulation of supersonic flow past a spike-tipped blunt nose.” Chinese Journal of Aeronautics 31, no. 9, (September 2018): 1815-1821. doi:10.1016/j.cja.2018.06.016.

[45] Anbu Serene Raj, C., M. Narasimhavaradhan, N. Vaishnavi, S. Arunvinthan, A. Al Arjani, and S. Nadaraja Pillai. "Aerodynamics of ducted re-entry vehicles.” Chinese Journal of Aeronautics 33, no. 7, (July 2020): 1837-1849. doi:10.1016/j.cja.2020.02.019.

[46] Akimov, G.A., D.O. Vavilov, and A.V. Klochkov. "Numerical study of supersonic jets of complex spatial configuration. Rocket Engineering." Misselery.info (2021). Available online: https://missilery.info/article/chislennoe-issledovanie-sverhzvukovyhstruy-slozhnoy-prostranstvennoy-konfiguracii (accessed on April 2021).

[47] Varsegov, Vladislav Lvovich. "Methodology of Designing the Thrust Reversal Device of a Bypass Jet Based on Mathematical and Numerical Modeling of Flow Aerodynamics" (2018).

[48] Kovalenko, N.D., G.A. Strelnikov, and A.E. Zolotko. "Gas-dynamic aspects and design of nozzles of rocket stage motors with the high-density layout." Technical Mechanics 3, (2011): 36-53.

[49] Vlasov, E.V., R.K. Karavosov, and V.F. Samokhin. “Acoustic field of a jet outflowing from a rectangular nozzle.”TsAGIScience Journal XXX, no. 1-2, (1999): 131-134. 